\title{
Universal correlations in pion-less EFT with the Resonating Group Method: three and four nucleons
}

\author{
Johannes Kirscher ${ }^{\star}$ and Harald W. Grießhammer \\ Center for Nuclear Studies, Department of Physics, \\ The George Washington University, Washington, DC 20052, USA \\ Deepshikha Shukla \\ Department of Physics and Astronomy, University of North Carolina, Chapel Hill, NC 27599-3255, USA \\ Hartmut M. Hofmann \\ Institut für Theoretische Physik III, Department für Physik, \\ Universität Erlangen-Nürnberg, D-91058 Erlangen, Germany
}

(Dated: November 19, 2018)

\begin{abstract}
The Effective Field Theory "without pions" at next-to-leading order is used to analyze universal bound state and scattering properties of the 3- and 4-nucleon system. Results of a variety of phase shift equivalent nuclear potentials are presented for bound state properties of ${ }^{3} \mathrm{H}$ and ${ }^{4} \mathrm{He}$, and for the singlet S-wave ${ }^{3} \mathrm{He}$-neutron scattering length $a_{0}\left({ }^{3} \mathrm{He}-\mathrm{n}\right)$. The calculations are performed with the Refined Resonating Group Method and include a full treatment of the Coulomb interaction and the leading-order 3-nucleon interaction. The results compare favorably with data and values from AV18(+UIX) model calculations. A new correlation between $a_{0}\left({ }^{3} \mathrm{He}-\mathrm{n}\right)$ and the ${ }^{3} \mathrm{H}$ binding energy is found. Furthermore, we confirm at next-to-leading order the correlations, already found at leadingorder, between the ${ }^{3} \mathrm{H}$ binding energy and the ${ }^{3} \mathrm{H}$ charge radius, and the Tjon line. With the ${ }^{3} \mathrm{H}$ binding energy as input, we get predictions of the Effective Field Theory "without pions" at next-toleading order for the root mean square charge radius of ${ }^{3} \mathrm{H}$ of $(1.6 \pm 0.2) \mathrm{fm}$, for the ${ }^{4} \mathrm{He}$ binding energy of $(28 \pm 2.5) \mathrm{MeV}$, and for $\operatorname{Re}\left\{a_{0}\left({ }^{3} \mathrm{He}-\mathrm{n}\right)\right\}$ of $(7.5 \pm 0.6)$ fm. Including the Coulomb interaction, the splitting in binding energy between ${ }^{3} \mathrm{H}$ and ${ }^{3} \mathrm{He}$ is found to be $(0.66 \pm 0.03) \mathrm{MeV}$. The discrepancy to data of $(0.10 \mp 0.03) \mathrm{MeV}$ is model independently attributed to higher order charge independence breaking interactions. We also demonstrate that different results for the same observable stem from higher order effects, and carefully assess that numerical uncertainties are negligible. Our results demonstrate the convergence and usefulness of the pion-less theory at next-to-leading order in the ${ }^{4} \mathrm{He}$ channel. We conclude that no 4-nucleon interaction is needed to renormalize the theory at next-to-leading order in the 4-nucleon sector.
\end{abstract}

PACS numbers: 21.45.-v, 21.45.Ff, 25.10.+s, 25.40.Dn, 27.10.+h

Keywords: Effective Field Theory; few-nucleon system; few-nucleon interactions; Resonating Group Model; universal correlations; ${ }^{3}$ He-neutron scattering length; charge symmetry breaking

\section{INTRODUCTION}

Nuclear processes, both with and without external probes, at energies well below the pion production threshold are naturally described by the Effective Field Theory "without pions" EFT (see e.g. [1 [ 5 ] for reviews). It shares with QCD the symmetries and effective low-energy degrees of freedom and provides a simple, systematic, and model independent approach appropriate for systems at very low energies. Like all Effective Field Theories, it allows for a systematic expansion of the scattering amplitude in a small, dimensionless parameter to attain the desired level of accuracy in observables and therefore provides reliable a priori error estimates. Its simple structure makes it also easier to handle than pionful EFT [6] [8].

$\mathrm{EFT}_{\star}$ has successfully been applied to many reactions of two and three nucleons with and without electro-weak currents. While calculations at fourth order have been employed to yield very accurate results in those systems (see e.g. [9]), higher order calculations involving more than three nucleons are still in their infancy. This work supplements the few, already existing leading-order (LO) calculations in the bound four-nucleon system [10, 11] by a next-toleading order (NLO) analysis including the Coulomb interaction and the leading-order 3-nucleon interaction. As first observable in the four-nucleon scattering system, it adds the singlet S-wave 3-Helium-neutron scattering length

${ }^{*}$ Corresponding author; electronic address: kirscher@gwu.edu 
$a_{0}\left({ }^{3} \mathrm{He}-\mathrm{n}\right)$. By that, it addresses the question whether $\mathrm{EFT}_{\not \rightarrow}$ still converges for the $\alpha$-particle.

Our investigation has three central goals: (i) to support evidence that the four-nucleon bound- and scattering system can be described by EFT ; (ii) to provide strong evidence that no four-nucleon-interaction is necessary to renormalize $\mathrm{EFT}_{\text {t }}$ at NLO; and most importantly (iii) to clearly demonstrate the feasibility of the variational approach of the "RRGM" (RRGM) for EFT in addressing these goals also for future studies involving heavier systems.

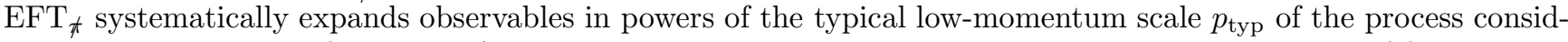
ered, measured in units of the scale $\Lambda_{\mathrm{EFT}_{\star}}$ at which the pion can be resolved as a dynamical degree of freedom, set by the pion mass $m_{\pi}$. Equivalently, the expansion parameter is given by the ratio of the range $r_{\pi} \approx 1.4 \mathrm{fm}$ of the one-pion-exchange over typical resolutions or sizes of the process under consideration. The 4-Helium binding energy is $28.5 \mathrm{MeV}$ and its typical size of about $1.6 \mathrm{fm}$ is comparable with the approximate range of the one-pion-exchange. A systematic expansion in the four-nucleon system could therefore converge very slowly at best. However, Platter et al. 10] showed that $\mathrm{EFT}_{\text {a }}$ at $\mathrm{LO}$ can explain with good accuracy the correlation between the 4-Helium and triton binding energies, the famous Tjon line [12]. One focus of this article is to test whether or not EFT at NLO shows a convergence order-by-order, and converges to experimental values.

For this purpose, we investigate correlations between three- and four-nucleon observables. They correspond to universality classes of nuclear interaction models, all resulting in on-shell (phase shift) equivalent 2-nucleon potentials which share the same two-body scattering lengths $a_{s, t}$ in the singlet and triplet $S$-wave. The elements of a class, parameterized by $a_{s, t}$, therefore fully describe the nucleon-nucleon (NN) system in the zero energy limit. In contradistinction, nonuniversal parameters like the effective range only add perturbative corrections and correspond to higher order interactions in the EFT power-counting. Their contribution to an observable vanishes in the zero energy limit. The empirically found Phillips [13] line, the Efimov spectrum of three-body bound states and the Tjon line [12] are examples for universal properties of NN potentials of the same universality class defined by $a_{s, t}$ (for reviews see e.g. [4, [5, 14]). The Phillips line is a correlation between the triton binding energy $B(t)$ and the neutron-deuteron doublet scattering length and can be explained as a result of the fact that on-shell equivalent 2-nucleon potentials have in general different off-shell behavior. Such a dependence of low-energy observables on details of short-distance 2-nucleon physics is however unphysical. This renormalization defect can be corrected by including a three-body interaction which eliminates the dependence on the NN potential. The datum on the Phillips line is then reproduced at LO by fitting the strength of the three-body interaction [15, 16]. In the zero energy limit, the 3-nucleon system is hence described by three universal parameters: $a_{s, t}$ and one three-body low-energy datum. Different values for this low-energy datum result in a different strength of the 3-nucleon interaction. As the latter is however also dependent on the NN potential used, one can use an alternative approach to explore the dependence of few-nucleon observables on the additional three-body input: Set the three-body interaction to zero and map out the dependence of observables on the regularization procedure used for the 2-nucleon potential. The results are correlations between few-body observables. In this work, we employ all three procedures to map out correlations: different values of the regulating cutoff, a variation of unresolved short-distance observables by allowing for non-zero, higher order P-wave and SD interactions, and a variation of the coupling strength of the three-body counterterm with fixed 2-nucleon interaction.

One might be tempted to choose form and value of the cutoff for the 2-nucleon potential such that the triton binding energy is reproduced "exactly", and then perform all calculations with such a highly fine-tuned potential, see e.g. [17]. However, such an approach would deprive one of a simple procedure to find an estimate of the theoretical uncertainty of the calculation. Including electromagnetic interactions will also be more cumbersome, as these form-factors have to be gauged and additional, transverse currents need to be constructed. On top of that, it is not clear that one can find such a 2-nucleon potential to eliminate any 3-nucleon interaction, or that its choice would be unique [64]. The numerical advantage of not having to deal with 3-nucleon interactions in some systems can in general be balanced by the problem to find the "right" 2-nucleon potential. In any case, such additional effort does not increase the reliability of the calculation. We therefore keep the cutoff arbitrary to map out correlations and error estimates.

Adding one more nucleon, the Tjon line of a one-parameter correlation between the binding energies of the triton and 4-Helium supports the assertion that no additional universal parameter is necessary to classify the four-nucleon system. Its emergence in $\mathrm{EFT}_{\text {a }}$ at $\mathrm{LO}$ was demonstrated by Platter et al. [10]. In order to extend this finding to NLO, this work investigates not only the dependence of the triton charge radius, the splitting in the trinucleon binding energies, and the 4-Helium binding energy on the triton binding energy, but also finds a similar connection between the real part of $a_{0}\left({ }^{3} \mathrm{He}-\mathrm{n}\right)$ and the triton binding energy. Therefore, it supports the expectation, based on naïve dimensional analysis, that no four-body interaction is necessary to renormalize the theory at NLO in the four-body sector. Up to this level of accuracy, observables in the four-nucleon system are universal consequences of the two- and three-body dynamics of its constituents. For all observables we consider, namely the charge radius of the triton, the trinucleon binding energy splitting, the 4-Helium binding energy $B(\alpha)$ and the scattering length $a_{0}\left({ }^{3} H e-n\right)$, we find predictions consistent with experiment within error margins when the 3-nucleon interaction strength is fitted to $B(t)$.

For this work, the Refined Resonating Group Method [18] is employed. This variational method numerically solves the Schrödinger equation in coordinate representation. Within a model space spanned by Gaussian type 
wave functions, minimization of a respective functional yields scattering and bound state observables for a potential parameterized in terms of Gaussians. With the RRGM, speedy scattering calculations are feasible in the $A=4$ system and even beyond, because of the relatively simple structure of the NLO EFT th potentials. To study the feasibility of using $\mathrm{EFT}_{\not \rightarrow}$ in the RRGM is the other focus of this article. In the course of this investigation, it is also imperative to carefully assess that the spread of results does not come from numerical inaccuracies.

The article is organized as follows: First, a brief overview of $\mathrm{EFT}_{\not \nless}$ is given as a low-energy theory which allows for a systematic improvement of the interaction amongst nucleons up to the desired accuracy. An introduction to the calculational tool of the RRGM in sect. IIII is followed by the derivation, structure, and fit of the NN potential in sect. IV The results are presented in sect. V] followed by a concluding section. An appendix addresses the numerical accuracy and computational costs of the calculations.

\section{PION-LESS THEORY}

In this section, the theoretical framework of an Effective Field Theory for nucleons without pions is recapitulated. For details, the reader is referred to the exemplary reviews [3, 19].

The nuclear potential is derived from a Lagrangean (see e.g. [20]) with contact interactions which are momentum independent at LO and momentum dependent at NLO:

$$
\begin{aligned}
\mathcal{L}_{\not k, N N}= & N^{\dagger}\left(i \partial_{0}+\frac{\nabla^{2}}{2 M}\right) N+C_{1}^{\mathrm{LO}}\left(N^{\dagger} N\right)\left(N^{\dagger} N\right)+C_{2}^{\mathrm{LO}}\left(N^{\dagger} \sigma_{i} N\right)\left(N^{\dagger} \sigma_{i} N\right) \\
& -\frac{1}{2} C_{1}^{\mathrm{NLO}}\left[\left(N^{\dagger} \partial_{i} N\right)^{2}+\left(\left(\partial_{i} N^{\dagger}\right) N\right)^{2}\right] \\
& -\left(C_{1}^{\mathrm{NLO}}-\frac{1}{4} C_{2}^{\mathrm{NLO}}\right)\left(N^{\dagger} \partial_{i} N\right)\left[\left(\partial_{i} N^{\dagger}\right) N\right]+\frac{1}{8} C_{2}^{\mathrm{NLO}}\left(N^{\dagger} N\right)\left[N^{\dagger} \partial_{i}^{2} N+\partial_{i}^{2} N^{\dagger} N\right] \\
& -\frac{i}{8} C_{5}^{\mathrm{NLO}} \epsilon_{i j k}\left\{\left[\left(N^{\dagger} \partial_{i} N\right)\left[\left(\partial_{j} N^{\dagger}\right) \sigma_{k} N\right]+\left[\left(\partial_{i} N^{\dagger}\right) N\right]\left(N^{\dagger} \sigma_{j} \partial_{k} N\right)\right]\right. \\
& \left.-\left(N^{\dagger} N\right)\left[\left(\partial_{i} N^{\dagger}\right) \sigma_{j} \partial_{k} N\right]+\left(N^{\dagger} \sigma_{i} N\right)\left[\left(\partial_{j} N^{\dagger}\right) \partial_{k} N\right]\right\}+\frac{1}{4}\left[\left(C_{6}^{\mathrm{NLO}}+\frac{1}{4} C_{7}^{\mathrm{NLO}}\right)\left(\delta_{i k} \delta_{j l}+\delta_{i l} \delta_{k j}\right)\right. \\
& \left.+\left(2 C_{3}^{\mathrm{NLO}}+\frac{1}{2} C_{4}^{\mathrm{NLO}}\right) \delta_{i j} \delta_{k l}\right]\left[\left[\left(\partial_{i} \partial_{j} N^{\dagger}\right) \sigma_{k} N\right]+\left(N^{\dagger} \sigma_{k} \partial_{i} \partial_{j} N\right)\right]\left(N^{\dagger} \sigma_{l} N\right) \\
& -\frac{1}{2}\left[C_{6}^{\mathrm{NLO}}\left(\delta_{i k} \delta_{j l}+\delta_{i l} \delta_{k j}\right)+C_{4}^{\mathrm{NLO}} \delta_{i j} \delta_{k l}\right]\left(N^{\dagger} \sigma_{k} \partial_{i} N\right)\left[\left(\partial_{j} N^{\dagger}\right) \sigma_{l} N\right] \\
& -\frac{1}{8}\left(\frac{1}{2} C_{7}^{\mathrm{NLO}}\left(\delta_{i k} \delta_{j l}+\delta_{i l} \delta_{k j}\right)-\left(4 C_{3}^{\mathrm{NLO}}-3 C_{4}^{\mathrm{NLO}}\right) \delta_{i j} \delta_{k l}\right) \\
& {\left[\left(\partial_{i} N^{\dagger} \sigma_{k} \partial_{j} N\right)+\left(\partial_{j} N^{\dagger} \sigma_{k} \partial_{i} N\right)\right]\left(N^{\dagger} \sigma_{l} N\right) . }
\end{aligned}
$$

The only effective low-energy degree of freedom is the nonrelativistic fermion iso-doublet $N=\left(\begin{array}{c}p \\ n\end{array}\right)$ of Weyl spinors $p$ and $n$ for the proton and neutron, respectively. In eqs. (12), Einstein's summation convention is understood, i.e., a sum from 1 to 3 over repeated Arabic indices. The Lagrangean is an isoscalar at this order, with neutrons and protons having the same mass $M$. The coupling constants $C_{i}^{(\mathrm{N}) \mathrm{LO}}$ are referred to as low-energy constants (LECs) or counterterms, and $\sigma_{i}$ are the Pauli spin matrices. The power-counting in EFT $\mathrm{E}_{\star}$ at NLO (see e.g. [2]) results in four independent LECs, with zero P-wave and SD-transition amplitudes providing the five constraining equations (see the discussion of eq. (11) for how this constraint is implemented).

Within the EFT framework, it was found [16, 21 25] that exactly one 3-nucleon contact interaction (3NI) is necessary to renormalize the $A=3$ system in the doublet-S channel at LO and NLO. In our NLO calculation, this three-body counterterm has the form

$$
\mathcal{L}_{t, 3 N}=C_{3 \mathrm{NI}}^{\mathrm{LO}}\left(N^{\dagger} N\right)\left(N^{\dagger} \tau_{i} N\right)\left(N^{\dagger} \tau_{i} N\right)
$$

Other forms are identical after Fiertz-transformations [22]. Once the 2-nucleon parameters in eq. (11) are fixed, the universal correlation lines between three- and four-nucleon observables, discussed in sect. $\mathrm{V}$, are parameterized by the 3-nucleon coupling $C_{3 \mathrm{NI}}^{\mathrm{LO}}$.

The amplitudes derived from the Lagrangean in eq. (1) are given as an expansion in the dimensionless parameter $Q \sim p_{\text {typ }} / \Lambda_{b}$, where $p_{\text {typ }}$ is a typical low-momentum scale of the system, and a rough estimate for the breakdown scale $\Lambda_{b}$ of the theory is the pion mass. In the neutron-proton system at center of mass energies of less than the deuteron 
binding energy $B(d), p_{\text {typ }}$ is set by the binding momentum, $\sqrt{M B(d)}$, which leads to an expansion parameter of $Q \lesssim \frac{1}{3}$. A calculation at $\mathrm{N}^{n} \mathrm{LO}$ is then expected to be accurate up to perturbative corrections of order $Q^{n+1}$. For energies below $B(d)$, the parameter $Q$ is found to be approximately constant (e.g. 26]) but increases for higher energies. This increase leads eventually to a breakdown of the perturbative expansion. In practice, a cornucopia of EFT $_{\text {\# }}$ calculations $[3,29,22,27,28]$ to higher orders has shown that the expansion converges somewhat beyond the pion mass and that $\frac{1}{5} \lesssim Q \lesssim \frac{1}{3}$.

The NN interaction is understood as an effective potential following Weinberg's original definition [29]. In the pion-less theory, the potential reduces to tree diagrams of two in- or out-going nucleons, with vertices from eq. (11). While not necessary from the EFT standpoint, it is convenient for this work to insert the full NLO potential in this form into the Schrödinger equation instead of treating the non-leading terms in perturbation theory. This course of action has been pursued regularly and includes some contributions which are formally of higher order in the EFT power-counting but does not increase the accuracy of the result. The re-summation does however lead to spurious bound states in the two-particle sector which can impact three-particle observables, see e.g. [28].

This method also allows us to briefly comment on how our results would change in an alternative power-counting in $\mathrm{EFT}_{\not \text { }}$ proposed by Beane and Savage [30], where both scattering lengths and effective ranges count as $1 / p_{\text {typ }} \sim Q^{-1}$. In that case, effective-range corrections must be re-summed, and some combinations of the NN interactions $C_{1-7}^{\mathrm{NLO}}$ in eq. (11) are promoted to LO. One would now be compelled to iterate the potential in the Schrödinger equation. As one performs technically the same steps as above, one arrives at the same amplitude. Our results can thus also be interpreted as LO calculations in the alternative formulation. The difference between this alternative and the approach taken here is therefore only in the question whether re-summing effective range contributions is optional or mandatory. If the effective ranges can but do not have to be included as NLO corrections, one expects that observables change only by parametrically small amounts when one calculates first with zero effective ranges (i.e. keeping $C_{1,2}^{\mathrm{LO}}$ only), and then adds the supposed NLO terms $C_{1-7}^{\mathrm{NLO}}$. Our results for the triton charge radius in sect. $\mathrm{VA}$ and for the Tjon line in sect. $\mathrm{VC}$ confirm this assumption in the three- and four-nucleon system. From that perspective, we therefore see no reason to make the effective-range resummation mandatory.

As pointed out in [31], for a short ranged potential like this, the Wigner bound can potentially constrain the value of the effective ranges: When the scattering length is positive, an energy-independent potential exists only below a cutoff-dependent upper bound for the effective range. For high enough cutoffs, the physically observed effective range in the ${ }^{3} S_{1}$ channel will exceed this Wigner bound. However, we will demonstrate in sect. [V] that for our choice of cutoffs, the problem does not arise.

The derivation of the explicit form of the potential in coordinate space from eq. (1) is postponed to sect. IV in favor of first introducing the numerical method used, as it motivates also the choice of operator structure for the potential.

\section{RESONATING GROUP METHOD}

We employ the variational method of the Refined Resonating Group Method (RRGM) [18] to solve the Schrödinger equation in coordinate space. In this section, the method is introduced. Factors determining the numerical stability of $\mathrm{EFT}_{\star}$ calculations with the RRGM are addressed in app. A.

The RRGM uses a Gaussian basis to span the variational space and a Gaussian expansion of the radial dependences of the nuclear potential which allow for an analytic calculation of the Hamilton matrix. As in every variational approach, the basis is incomplete. Care has to be taken to avoid linearly dependent basis vectors. The computer time for the calculation depends on the dimension of the variational space, the number of Gaussians needed for an accurate fit of the radial functions of the potential, and its operator structure. A Gaussian regulator for the contact interactions leads directly to a Gaussian radial dependence of the potential and makes the RRGM an efficient tool to analyze the few-nucleon sector. This section describes those aspects of the method which are necessary to understand the choices made for the form of the regulator function, for the range of its cutoff values, and for the set of operators which constitute the potential.

To determine the bound state wave function, the Ritz functional is minimized in a model space spanned by vectors of the form

$$
\psi_{\mathrm{BS}}^{J^{\pi}}\left(\vec{\rho}_{m}, \vec{s}_{m}\right)=\mathcal{A}\left\{\sum_{d, i, j} c_{d i j}\left[\left[\prod_{k=1}^{N-1} e^{-\gamma_{d k} \vec{\rho}_{k}^{2}} \mathcal{Y}_{l_{k i}}\left(\vec{\rho}_{k}\right)\right]^{L_{i}} \otimes \Xi^{S_{j}}\right]^{J} \cdot \Upsilon\right\}
$$

The system consists of $N$ particles. To each of the $N-1$ Jacobi coordinates $\vec{\rho}_{k}$, one assigns a set of different width parameters $\gamma_{d k}$ and of different angular momenta $l_{k i}$ represented by solid spherical harmonics $\mathcal{Y}_{l m}$ [32]. The antisymmetrizer is denoted by $\mathcal{A}$, and the square brackets indicate angular momentum couplings, with the orbital 
part $L_{i}$ being combined with the spin $S_{j}$ to the total angular momentum $J$. The label $d$ distinguishes different sets of width parameters $\gamma$, while $i$ and $j$ label sets of orbital- and spin angular momentum coupling schemes. The spin function $\Xi$ is constructed as a sum of products of the single particle spin functions $\vec{s}$. The isospin function $\Upsilon$ is built analogously from single particle isospin functions and distinguishes between neutrons and protons. The superposition coefficients $c_{d i j}$ are determined by minimizing the Ritz functional. The magnetic quantum numbers of the spherical tensors are not explicit in the above equation where they are not needed to label a specific basis vector. Since one deals only with reduced matrix elements, even the magnetic quantum number corresponding to the total angular momentum $J$ is of no significance.

For the scattering state, the Kohn-Hulthén variational principle is used with the following ansatz for the wave function:

$$
\begin{aligned}
\psi_{\mathrm{SS}, \lambda}^{J^{\pi}}= & \mathcal{A}\left\{\sum_{j}^{n_{k}}\left[\frac{1}{R_{j}} Y_{L_{j}}\left(\hat{\vec{R}}_{j}\right) \otimes\left[\psi_{j}^{J_{1}^{\pi_{1}}} \otimes \psi_{j}^{J_{2}^{\pi_{2}}}\right]^{S_{c_{j}}}\right]^{J} .\right. \\
& \left.\left(\delta_{\lambda j} F_{L_{j}}\left(R_{j}\right)+a_{\lambda j} \tilde{G}_{L_{j}}\left(R_{j}\right)+\sum_{m} b_{\lambda j m} R_{j}^{L_{j}+1} e^{-\omega_{j m} \vec{R}_{j}^{2}}\right)\right\} .
\end{aligned}
$$

The two fragments are represented by bound state wave functions $\psi_{j}^{J_{1,2}^{\pi_{1}, 2}}$, determined by the aforementioned Ritz minimization and built from vectors as in eq. (3). The scattered fragments are separated by $\vec{R}$, and the orbital angular momentum between the two fragments is carried by a spherical harmonic $Y_{L}$. $\lambda$ specifies the boundary condition which allows regular Coulomb waves $F_{L}$ [33] only for the channel $j=\lambda$. The reactance coefficients $a_{\lambda j}$ and the $b_{\lambda j m}$ are determined by minimizing the Kohn-Hulthén functional, taking into account $n_{k}$ channels. The additional set of variational parameters $b_{\lambda j m}$ is necessary to approximate the wave function in the interaction region. The irregular Coulomb functions are regularized with a polynomial weighted by an exponential and renamed $\tilde{G}_{L}$. To become more familiar with the terminology and as a precursor to the calculation presented in sect. V] consider a neutron scattered off a 3 -Helium nucleus. In that case, $n_{k}=654$ channels were included, the fragment wave function of the neutron is $\psi_{(n)}^{\frac{1}{2}^{+}}=1$, and that of the 3 -Helium $\psi_{\left({ }^{\frac{1}{2}} \mathrm{He}\right)}^{+}$. The latter consisted of 224 terms, namely 78 $\left(L_{1}=0, L_{2}=0\right)$ components, called $S S$-configuration, $82 S D-, 45 D D-$, and $21 P P$-configurations. An example of an SD-configuration might be given by the following parameters: The angular momenta $l_{1}=0, l_{2}=2$ on the two Jacobi coordinates couple to a total orbital angular momentum $L=2$. This mandates the individual spins of the nucleons to be aligned, i.e., total spin $S=\frac{3}{2}$, so that finally a part of the triton system with $J_{2}^{\pi}=\frac{1}{2}^{+}$is formed. The two total angular momenta of the fragments, $J_{1,2}^{\pi}=\frac{1}{2}^{+}$, can be coupled to channel spins, $S_{c} \in\{0,1\}$. However, only $S_{c}=0$ has to be included for the $J^{\pi}=0^{+}$-channel, which in turn dictates that the orbital angular momentum between the fragments is zero, $L_{j}=0$. The width parameter $\omega_{j m}$ for the inter-fragment wavefunction plus two widths $\gamma_{d k}$ for the 3-Helium bound state component complete the specification of the basis vector. In the following, the model space is defined to be a vector space spanned by basis vectors $\psi_{\mathrm{BS} / \mathrm{SS}}$ as given in eq. (3) or eq. (4).

Once the potential is written in terms of spherical tensor operators and its radial dependences are expressed in Gaussian functions, all the coordinate space matrix elements which are needed to minimize the respective functional can be cast into the form

$$
I=\int d^{3} \rho_{1} \ldots d^{3} \rho_{n_{k}-1} \exp \left[-\sum_{\nu \nu^{\prime}}^{n_{k}-1} C^{\nu \nu^{\prime}} \vec{\rho}_{\nu} \cdot \vec{\rho}_{\nu^{\prime}}+\sum_{\nu}^{n_{l}} \vec{\xi}^{\nu} \cdot \vec{\rho}_{\nu}\right] .
$$

The matrix $C^{\nu \nu^{\prime}}$ transforms the Jacobi coordinates in the in-coming channel and the relative coordinates of the radial dependences in the potential operator to the coordinates in the out-going channel. Furthermore, it takes into account that the antisymmetrizer permutes single particle coordinates. The vector $\vec{\xi}^{\nu}$ is related to the generating function of spherical harmonics. Its dimension is $n_{l}$, namely the number of spherical harmonics in the matrix element $I$. The integrals $I$ can hence be evaluated analytically for all operators of the EFT

For an accurate description of the scattering state, so-called distortion channels have to be added to the physical channels in eq. (4). They increase the variational space to allow for a more accurate description of the wave function in the interaction region. There, a separation into two bound fragments as mimicked by the physical channels does not resemble, e.g. three- or four-body breakup states. Those channels do not have an asymptotic tail, i.e., only the square integrable terms with coefficients $b_{\lambda j m}$ in eq. (4) constitute the relative part of their wave function. Furthermore, their fragment functions are not restricted to describe bound states, but only to have the correct quantum numbers. That means it suffices to specify a single set $\left\{l_{k i}, \gamma_{d k}, S_{j}\right\}$, instead of superimposing multiple sets to a bound state. In the calculations, all but one of the components of each of the physical channels were used for this purpose, e.g., a 
physical 3-Helium-neutron channel, with 224 basis vectors for the 3-Helium fragment, yields 223 configurations. The number of distortion channels, and by that the dimension of the model space, is ultimately determined by the number of different $\omega_{j m}$ used in eq. (4). With the number of physical channels fixed, more distortion channels were added to obtain a converged result for the observables.

In view of the error analyses performed in sect. $\mathrm{V}$, it is imperative to demonstrate that purely numerical inaccuracies of the variational method are no significant source of error. In app. A, we demonstrate that differences in calculations of the same observable do indeed not originate from numerical inaccuracies.

\section{TWO-NUCLEON POTENTIAL AND PARAMETER DETERMINATION}

In this section, the potential in coordinate representation is derived from the Lagrangean in eq. (1), following the lines of [20] but choosing a different operator structure which simplifies the implementation into the RRGM. In the second part, the fitting procedure of the low-energy constants (LEC) and the experimental input is explained.

In momentum representation, the two-body potential for an $A$-nucleon system following from the nine four-nucleon contact interactions of eq. (1) is

$$
\begin{aligned}
V_{\mathrm{EFT}_{*}, N N}^{(N L O)}=\sum_{i<j}^{A}( & C_{1}^{\mathrm{LO}}+C_{2}^{\mathrm{LO}} \vec{\sigma}_{i} \cdot \vec{\sigma}_{j}+C_{1}^{\mathrm{NLO}} \vec{q}^{2}+C_{2}^{\mathrm{NLO}} \vec{k}^{2}+\vec{\sigma}_{i} \cdot \vec{\sigma}_{j}\left(C_{3}^{\mathrm{NLO}} \vec{q}^{2}+C_{4}^{\mathrm{NLO}} \vec{k}^{2}\right) \\
& \left.+i C_{5}^{\mathrm{NLO}} \frac{\left(\vec{\sigma}_{i}+\vec{\sigma}_{j}\right)}{2} \cdot \vec{q} \times \vec{k}+C_{6}^{\mathrm{NLO}} \vec{q} \cdot \vec{\sigma}_{i} \vec{q} \cdot \vec{\sigma}_{j}+C_{7}^{\mathrm{NLO}} \vec{k} \cdot \vec{\sigma}_{i} \vec{k} \cdot \vec{\sigma}_{j}\right)
\end{aligned}
$$

with $\vec{q}=\vec{p}-\vec{p}^{\prime} \quad, \quad \vec{k}=\frac{\vec{p}+\vec{p}^{\prime}}{2}$ defined in terms of the in(out)going center of mass momenta $\vec{p}\left(\vec{p} \vec{p}^{\prime}\right)$ of one nucleon. Following eq. (2), the three-body potential is given as

$$
V_{\mathrm{EFT}_{\star}, 3 N}^{(L O)}=\sum_{i<j<k}^{A} C_{3 \mathrm{NI}}^{\mathrm{LO}}\left(\vec{\tau}_{i} \cdot \vec{\tau}_{j}+\vec{\tau}_{k} \cdot \vec{\tau}_{i}+\vec{\tau}_{j} \cdot \vec{\tau}_{k}\right)
$$

Regularized with $f_{\Lambda}(\vec{q})=\exp \left(-\vec{q}^{2} / \Lambda^{2}\right)$ and Fourier transformed, eq. (6) is cast into the form

$$
\begin{aligned}
V_{\mathrm{EFT}_{\sharp}, N N}^{(N L O)}= & \sum_{i<j}^{A} I_{0}(\Lambda, r)\left(A_{1}+A_{2} \vec{\sigma}_{i} \cdot \vec{\sigma}_{j}\right)+\left(A_{3}+A_{4} \vec{\sigma}_{i} \cdot \vec{\sigma}_{j}\right)\left\{I_{0}(\Lambda, r), \vec{\nabla}^{2}\right\}+ \\
& I_{0}(\Lambda, r)\left(A_{5}+A_{6} \vec{\sigma}_{i} \cdot \vec{\sigma}_{j}\right) \vec{r}^{2}+ \\
& I_{0}(\Lambda, r) A_{7} \vec{L} \cdot \vec{S}+I_{0}(\Lambda, r) A_{8}\left(\vec{\sigma}_{i} \cdot \vec{r} \vec{\sigma}_{j} \cdot \vec{r}-\frac{1}{3} \vec{r}^{2} \vec{\sigma}_{i} \cdot \vec{\sigma}_{j}\right) \\
& -A_{9}\left\{I_{0}(\Lambda, r),\left[\left[\partial^{r} \otimes \partial^{s}\right]^{2} \otimes\left[\sigma_{1}^{p} \otimes \sigma_{2}^{q}\right]^{2}\right]^{00}\right\},
\end{aligned}
$$

and eq. (7) into

$$
V_{\mathrm{EFT}_{\sharp}, 3 N}^{(L O)}=\sum_{\substack{i<j<k \\ \text { cyclic }}}^{A} I_{0}\left(\Lambda, r_{i j}\right) I_{0}\left(\Lambda, r_{j k}\right) C_{3 \mathrm{NI}}^{\mathrm{LO}} \vec{\tau}_{i} \cdot \vec{\tau}_{j}
$$

with the interparticle vector $\vec{r}=\vec{r}_{i}-\vec{r}_{j}$, the orbital angular momentum operator $\vec{L}=-i \vec{r} \times \vec{\nabla}$, and total spin operator $\vec{S}=\frac{1}{2}\left(\vec{\sigma}_{i}+\vec{\sigma}_{j}\right)$, as well as the regulator function $I_{0}(\Lambda, r)=\exp \left(-\Lambda^{2} \vec{r}^{2} / 4\right)$. It is not necessary to symmetrize the potential with respect to particle exchange because the basis states are antisymmetric. Instead of fitting the original LECs $C_{i}$, it was favorable to adjust directly linear combinations

$$
A_{i}=\sum_{j=1}^{9} a_{i j} \Lambda^{n(j)} C_{j}
$$

This re-definition avoids a fine-tuning of large versus small terms in the sum which arises because the anticommutators absorb powers of $\Lambda$ into the derivative acting on the regulator function $I_{0}(\Lambda, r)$.

The operator sets of AV18 and CD-Bonn are subsets of the one of eq. (8), while the relatively complicated radial dependences of the former contrasts with the polynomial-weighted Gaussians of eq. (8). Although most of the operator 
structure is hidden in the anti-commutators, Hermitecity is manifest in this form, and the Gaussian radial dependences allow for efficient RRGM calculations. The natural size of the coefficients $A_{i}$ is not trivial to estimate because the derivatives in the two anticommutators act not only on the wave function but also on the Gaussians in the potential. Therefore, the parameters $A_{3,4,9}$ are of lower power in $\Lambda$ and hence differ considerably in size relative to the others.

The NN P-wave amplitudes are $\mathrm{N}^{3} \mathrm{LO}$ in $\mathrm{EFT}_{\not \nmid}$. Hence, four constraints,

$$
\left\langle{ }^{2 S+1} P_{J}\left|V_{\mathrm{EFT}_{\star}, N N}^{(N L O)}\right|^{2 S+1} P_{J}\right\rangle=0
$$

can be employed to reduce the number of parameters to five. Here, we do not impose those constraints exactly, but instead extend the $\chi^{2}$-measure to fit the P-wave phase shifts to a fraction of the Nijmegen values,

$$
\delta_{\text {fit }}\left({ }^{2 S+1} P_{J}\right) \leq 0.1 \delta_{\mathrm{Nij}}\left({ }^{2 S+1} P_{J}\right)
$$

This is fully consistent with the EFT philosophy that higher order interactions can only induce higher order corrections in observables. Different P-wave constraints, compatible with eq. (12), allow for such a controlled modification of short-distance structure. An exact implementation of eq. (11) provides no significant gain in computer time relative to eq. (12). We therefore exploit the additional handle on higher order effects provided by eq. (12) to gauge the accuracy of a NLO calculation. A consequence of this approach are non-vanishing rank one and two interactions corresponding to LECs $A_{7}$ and $A_{8,9}$, respectively. These tensor structures are also found when one implements the SD-interactions, which enter beyond NLO. We choose to include these as representations of higher-order effects and to constrain their parameters by the SD-mixing angle.

Different sets of weight factors for the phase- and $B(d)$-deviations were used for the various potentials. Therefore, this $\chi^{2}$ is no objective criterion for the quality of the potential and we abstain from quoting it.

The basis of this potential does not mix the singlet with the triplet NN channels. Therefore, it was convenient to fit the projections of the potential in the spin singlet- and triplet channels separately. In the former, three parameters were adjusted to reproduce $\delta_{\mathrm{Nij}}\left({ }^{1} S_{0}\right)$ and $\delta_{\text {fit }}\left({ }^{1} P_{1}\right)$. In the triplet channel, the fit is initially to $B(d)$ only, followed by a fine-tuning of the six LECs to refine $\delta_{\text {fit }}\left({ }^{3} S_{1}\right), \delta_{\text {fit }}\left({ }^{3} P_{0,1,2}\right)$ and the SD-mixing angle $\epsilon_{1}$ fit $\left({ }^{3} S_{1}-{ }^{3} D_{1}\right)$. Here, the following hierarchy of weights $w$ (observable) in the $\chi^{2}$-function was used: $w(B(d))>w\left(\delta\left({ }^{3,1} S_{1,0}, \epsilon_{1}\right)\right)>w\left(\delta\left({ }^{2 S+1} P_{J}\right)\right)$, i.e. the most weight was put onto $B(d)$, and we typically used a ratio of $w(B(d)) / w(\delta)$ of the order of 10 . Dependence of the resulting values for the nine LECs on the model space was minimized by using an almost complete set of 40 Gaussian basis states as described in sect. III.

The $\chi^{2}$-minimization which determined the LECs was carried out with a modified version of the genetic search algorithm already used for wave function optimizations in [34]. This algorithm has the advantage of being independent from an educated guess for an initial set of parameters. With the terminology specified in [34], the search parameters for the algorithm were chosen as follows. The initial population was set to consist of more than $10^{4}$ individuals, with each individuum corresponding to a set of LECs. This relatively large number should ensure that a good fraction of the entire parameter space is probed. The search intervals used for the $A_{i}$ 's were chosen differently: $A_{3,4,9} \in\left[-10^{3} ;+10^{3}\right]$, while $A_{1,2,5-8} \in\left[-10^{4} ;+10^{4}\right]$. A factor of up to $\Lambda^{4}$ is not included in the $A_{3,4,9}$ but contributes when the derivatives in the anticommutator act on the regulator functions (see above). Therefore, we expect the $A_{3,4,9}$ to be smaller than the other $A_{i}$ 's.

The contribution a specific operator made to $B(d)$ differed amongst the ${ }^{i} V_{\not}$. While some EFT potentials distribute $B(d)$ similar to the potential models, AV18 [35] and CD-Bonn [36], i.e. the largest fractions coming from the central, and tensor terms, others summed $B(d)$ quite differently. However, there is no physical reason why one operator of ${ }^{i} V_{\not t}$ should contribute more than the other. Not only are LO and NLO operators combined in $V_{\mathrm{EFT}}^{\mathrm{NLO}}(\vec{r})($ see eq. (8) $)$, but they also have portions of the two central operators hidden in the central anticommutator, as well as a tensor component in the rank two anticommutator.

A heuristic explanation for the emergence of a variety of different LEC sets each representing a valid EFT $\mathrm{E}_{\not \rightarrow}$ interaction shall now be given. The internal structure of the nucleons is encoded in the LECs, and different regulators lead, after proper renormalization, to the same low-energy NN observables. Therefore, no unique set of coupling constants is expected. Here, two methods are employed to model different short-distance interactions with the same long distance behavior. First, the regulator was altered by changing the magnitude of the cutoff. Second, different sets of LECs were found by using different input for the fit.

The difference in input data must be compatible with the order at which the EFT calculation is carried out in the following sense. A consistent EFT calculation at order $n$ predicts low-energy observables accurately up to uncertainties of order $Q^{n+1}$ in the dimensionless expansion parameter. This property extents to the data used to fit the LEC, i.e. there is no need to refine the fit of the parameters in table I for a better reproduction of, e.g. the deuteron binding energy $B(d)$ of the potentials. By weighting data differently in the $\chi^{2}$-function, different sets of LECs were found, see table 【. The values for $B(d)$ and the neutron-proton phase shifts for those sets were all in the NLO uncertainty 
TABLE I: Numerical values of the cutoff $\Lambda$ and the LECs of the NLO EFT and NN phase shifts.

\begin{tabular}{|c|c|c|c|c|c|c|c|c|c|c|}
\hline & $\begin{array}{c}\Lambda \\
{[\mathrm{MeV}]}\end{array}$ & $\begin{array}{c}A_{1} \\
{[\mathrm{MeV}]} \\
\end{array}$ & $\begin{array}{c}A_{2} \\
{[\mathrm{MeV}]}\end{array}$ & $\begin{array}{c}A_{3} \\
{\left[\mathrm{MeV} \cdot \mathrm{fm}^{2}\right]}\end{array}$ & $\begin{array}{c}A_{4} \\
{\left[\mathrm{MeV} \cdot \mathrm{fm}^{2}\right]}\end{array}$ & $\begin{array}{c}A_{5} \\
{\left[\mathrm{MeV} \cdot \mathrm{fm}^{-2}\right]}\end{array}$ & $\begin{array}{c}A_{6} \\
{\left[\mathrm{MeV} \cdot \mathrm{fm}^{-2}\right]}\end{array}$ & $\begin{array}{c}A_{7} \\
{[\mathrm{MeV}]}\end{array}$ & $\begin{array}{c}A_{8} \\
{\left[\mathrm{MeV} \cdot \mathrm{fm}^{-2}\right]}\end{array}$ & $\begin{array}{c}A_{9} \\
{\left[\mathrm{MeV} \cdot \mathrm{fm}^{2}\right]}\end{array}$ \\
\hline 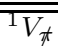 & $\overline{414}$ & $\overline{-143}$ & $\overline{464}$ & $\overline{-57.0}$ & $\overline{\overline{68.8}}$ & $\overline{-52.4}$ & $\overline{-346}$ & -3.97 & $\overline{-62.1}$ & $\overline{-0.111}$ \\
\hline${ }^{2} V_{\not t}^{\prime \prime}$ & 432 & -612 & 944 & -157 & 110 & 351 & -723 & 66.0 & -168 & -0.137 \\
\hline${ }^{3} V_{\text {t }}$ & 544 & -1224 & 1036 & -432 & 336 & 1704 & -1851 & -78.9 & -378 & -0.130 \\
\hline${ }^{4} V_{\not t}$ & 544 & -1625 & -89.6 & -298 & 49.8 & 1870 & 34.3 & -990 & -734 & $2.13 \cdot 10^{-3}$ \\
\hline${ }^{5} V_{\text {th }}^{\text {t }}$ & 544 & -405 & 317 & -125 & 108 & 287 & -493 & -271 & -360 & $-9.88 \cdot 10^{-4}$ \\
\hline${ }^{6} V_{\text {th }}^{\text {r. }}$ & 648 & 84.9 & -324 & -888 & 404 & 4845 & -2064 & -342 & -2319 & -0.716 \\
\hline 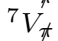 & 648 & -1316 & 1039 & -530 & 431 & 3775 & -3666 & -75.4 & -1052 & -0.178 \\
\hline${ }^{8} V_{\text {t }}^{\prime \prime}$ & 672 & 301 & -143 & -708 & 480 & 3527 & -3450 & -192 & -1613 & -0.257 \\
\hline${ }^{9} V_{t}$ & 672 & -158 & -201 & -362 & 275 & 2001 & -1659 & 79.7 & -1270 & -0.0850 \\
\hline
\end{tabular}

TABLE II: Two-nucleon observables calculated with NLO EFT potentials as defined in eq. (8) and table [1. The deuteron binding energies $B(d)$ are labeled by the RRGM model space in which they were calculated. The data for the scattering lengths $a_{s, t}$ and the effective ranges $r_{s, t}$ is taken from [38].

\begin{tabular}{|c|c|c|c|c|c|c|c|c|}
\hline & $\sum_{\Sigma}^{\overline{0}}$ & $\begin{array}{l}l_{d 20}^{w_{120}} \\
>_{0} \\
\sum\end{array}$ & 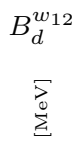 & $B_{d}^{w_{63}}$ & $\begin{array}{l}a_{s} \\
-1 \\
\vdots \\
\vdots \\
\vdots \\
\vdots\end{array}$ & $\begin{array}{l}a_{t} \\
-7 \\
1 \\
\vdots \\
\sum_{\Delta}\end{array}$ & 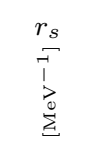 & $\begin{array}{l}r_{t} \\
\stackrel{-}{1} \\
\vdots \\
\stackrel{0}{ \pm}\end{array}$ \\
\hline$\overline{\mathrm{xpD}}$ & - & 2.225 & 2.225 & 2.225 & $\overline{0.120}$ & $\overline{.0275}$ & 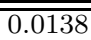 & $\overline{.00892}$ \\
\hline${ }^{1} V_{t}$ & 414 & 2.239 & 238 & 2.226 & .121 & 0275 & 0.0136 & 0.00914 \\
\hline${ }^{2} V_{t}^{\pi}$ & 432 & 224 & 2.212 & 2.203 & -0.119 & 0.0279 & 0.0134 & 104 \\
\hline${ }^{3} V_{t}^{\pi}$ & 544 & 221 & 2.205 & 2.153 & 23 & 268 & 0 & 824 \\
\hline${ }^{4} V_{t}$ & 544 & 233 & 2.201 & 2.185 & -0.119 & 0270 & 0 & 859 \\
\hline${ }^{5} V_{\not \star}^{n}$ & 544 & 224 & 2.222 & 2.186 & -0.119 & 0.0258 & 33 & 666 \\
\hline${ }^{6} V_{\pi}^{\pi}$ & 648 & 212 & 2.019 & 2.034 & -0.120 & 0.0271 & 132 & 835 \\
\hline${ }^{7} V_{t}$ & 648 & 2.214 & 2.137 & 2.012 & -0.115 & 0.0260 & 0.0124 & 0.00687 \\
\hline${ }^{8} V_{t}^{n}$ & 672 & 221 & 1.985 & 2.124 & -0.118 & 0.0264 & 0.0125 & 0.0077 \\
\hline${ }^{9} V_{t \pi}^{\pi}$ & 672 & 250 & 2.182 & 2.114 & -0.118 & 0.0255 & 0.0125 & 0.00648 \\
\hline
\end{tabular}

range around data, see table [II. Another approach which yields potentials with the same long-distance but different short-distance structure is changing the input for the $\mathrm{P}$-waves. The $\mathrm{P}$-wave phase shifts are observables of higher order, and therefore the values predicted by the NLO potentials are only restricted to a range compatible with zero. We enforce different $\mathrm{P}$-wave interactions by including and varying an appropriate term in the $\chi^{2}$-function, see eq. (12). We employed a combination of both methods, different weights and different P-wave input, to find several NN phase shift equivalent potentials for a single cutoff value.

Another point had to be kept in mind for the fit. In principle, the EFT philosophy allows for deeply bound states because reactions at energies for which the pion-less theory is applicable will not probe those low-lying, nonphysical states. As mentioned in sect. III, they can occur when re-summing effective range contributions. Nevertheless, we considered only potentials which were found not to posses any of these deeper lying bound states. The reason is that the variational basis does not span the entire Hilbert space and therefore might be insufficient to expand a low-lying and hence very localized ground state of a potential in the course of the fit. In that case, it would be impossible to tell if this state is low enough in energy to be considered marginal. To ensure that no such ghost states were present during the fit, vectors with narrower width parameters were added to the basis for the deuteron and the triton to model more localized wave functions and sort out the troublesome LEC parameter sets. Unphysical states might also be formed as clusters in a three- or four-nucleon calculation because of narrower basis states, in which case the corresponding LEC set was sorted out. We employed as figures of merit for a potential a converged value of the $\chi^{2}$-function after successive runs of the genetic algorithm, and the numerical stability of the deuteron and triton binding energies with respect to changes in the model space, see discussion below. The numerical values of the LECs over a range of cutoffs are presented in table \. The results for the deuteron binding energy in three different model spaces per potential are listed in table II to demonstrate the approximate closure of the set $w_{120}$ which was used to fit the LECs. A model space dependence of the potential would lead to different deuteron binding energies $B(d)$ in $w_{12}$ and $w_{120}$. Those sets were 40-dimensional, while the set $w_{63}$ is only 9-dimensional, with 6(3) optimized widths for the $\mathrm{S}(\mathrm{D})$ wave function component. It was used in the $A=4$ calculations to build the deuteron fragments. The change in $B(d)$ associated with the substitution of $w_{120}$ by $w_{12}$ increases with the cutoff. This is due to the absence of narrower width parameters in $w_{12}$ which, as seen above, has a greater impact for narrower potentials, or larger cutoffs, respectively. Hence, this change is attributed to a shortcoming of the $w_{12}$ model space rather than to 

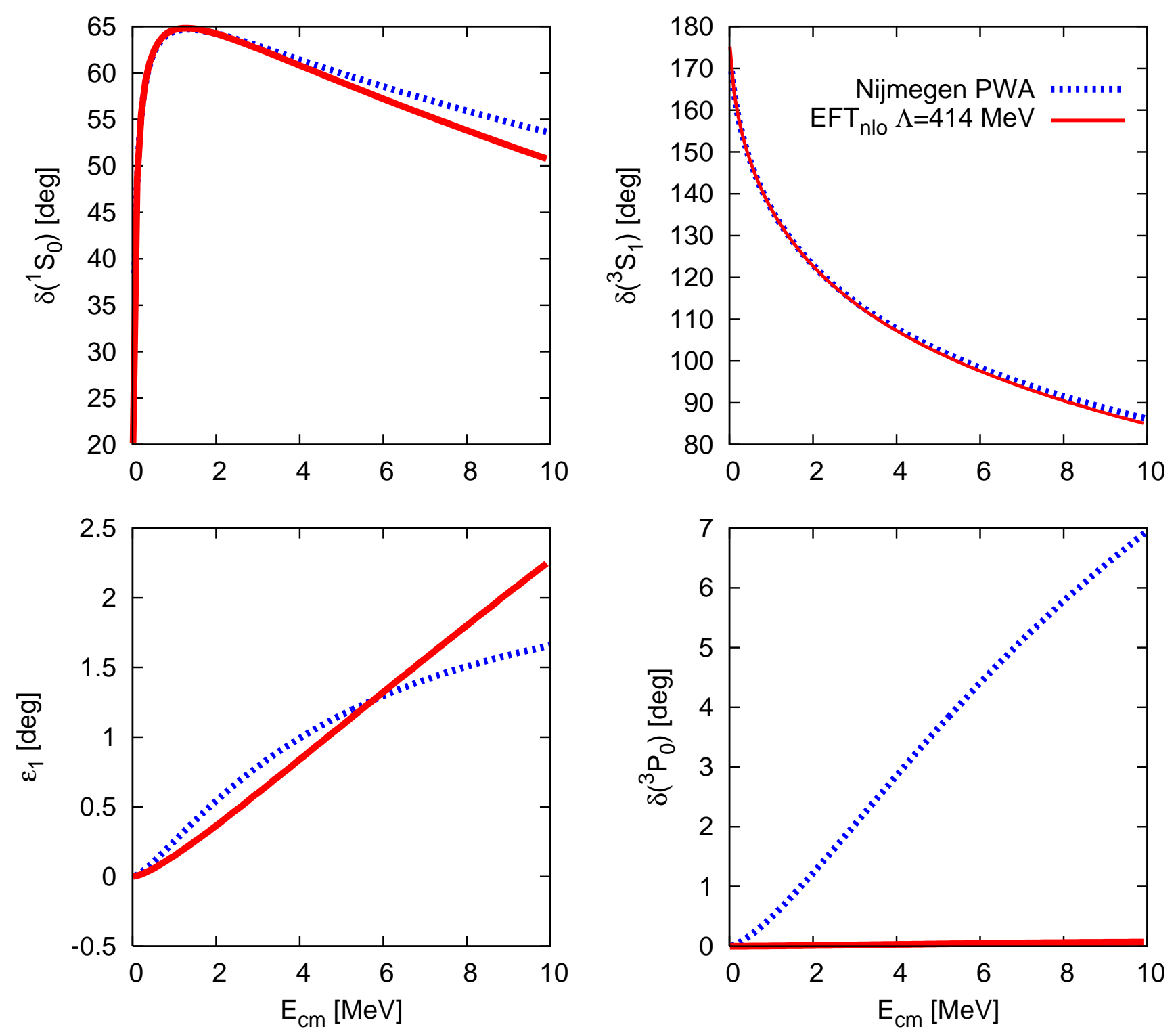

FIG. 1: Selected neutron-proton scattering phase shifts for the ${ }^{1} V_{\not \nless}$ potential and values from the Nijmegen partial wave analysis [39, 40] which were used to fit the LECs.

one of the potential. For the potential ${ }^{8} V_{\mathfrak{t}}, B(d)$ decreases considerably from $w_{120}$ to $w_{12}$ but increases again and approaches the $w_{120}$ value in a much smaller but optimized space $w_{63}$. In general, the quality of the model space used for the fitting procedure is comparable to the one used for the four-nucleon calculations presented in the next section.

The decrease in $B(d)$ when going from $w_{120}$ to $w_{63}$ has no substantial impact on the results for two reasons. First, $w_{63}$ was only used to expand the deuteron cluster in the 4-Helium system and not the one in the triton/3-Helium, which was built and optimized independently of the deuteron parameters. Second, in the $A=4$ system only scattering reactions at energies below the deuteron-deuteron threshold were considered. Therefore, the deuteron configurations contribute only through the distortion channels. This leads to the non-converged results for the imaginary part of the 4-nucleon scattering length, see sect. VD

The width parameters which constitute set $w_{63}$ were tailor-made for each potential using the genetic algorithm. In this process, the $w_{63}$ found for smaller cutoff values consisted on average of wider Gaussian width parameters compared to the ones for relatively large values of $\Lambda$. This behavior was already explained in sect. [IV in the discussion of fig. 6. While $B(d)$ was used as experimental input to determine the LECs, the singlet and triplet scattering lengths $a_{s, t}$ were used only implicitly through their dependence on the phase shifts in the respective channels. They were 
calculated from the appropriate S-matrix elements at a center of mass energy, $E_{\mathrm{cm}}=1 \mathrm{keV}$ (see discussion below, eq. (18) $)$.

As an example, the phase shifts for ${ }^{1} V_{\star}$ are shown in fig. 1 along with the phases from the Nijmegen analysis 39, 40]. Only phase shifts for a center of mass energy below $0.35 \mathrm{MeV}$ were used to determine the LECs, so that phases for higher energies can be used to gauge the quality of the fit. The other potentials ${ }^{2-9} V_{\not \star}$ reproduce the Nijmegen phases with the same accuracy, i.e., the phases up to $10 \mathrm{MeV}$ differ by less than $1 \%$. While $\delta_{\mathrm{Nij}}\left({ }^{1} S_{0}\right)$ and $\delta_{\mathrm{Nij}}\left({ }^{3} S_{1}\right)$ are reproduced up to $1 \%$ for $E_{\mathrm{cm}} \lesssim 10 \mathrm{MeV}$, the SD-mixing angle $\epsilon_{1}$ reaches, in the same energy interval, only a $10 \%$ accuracy and also showed a stronger variation between the various potentials. Both uncertainties are within the error margins predicted by $\mathrm{EFT}_{\not{t}}$, i.e., approximately $10 \%$ for the S-waves at typical momenta $p_{\text {typ }} \approx 45 \mathrm{MeV}$ and $30 \%$ for the higher-order observable $\epsilon_{1}$. For all potentials ${ }^{i} V_{\not}$ the results for the phase shifts in the ${ }^{3} D_{1}$ channel were close to zero, $\delta\left({ }^{3} D_{1}\right) \approx 0$. The ${ }^{3} P_{0}$ channel is displayed to exemplify that the $\mathrm{P}$-wave interactions of the potentials are indeed small.

Finally, observe in table Ihat our cutoff values do not constrain the effective range to unphysical values, as alluded to in the discussion of the Wigner bound in sect. II.

Table【and fig. 1demonstrate: (i) The quality of the fit is sufficient for a NLO calculation with the expected accuracy level of about $10 \%$ for $\mathrm{EFT}_{\not \text { }}$ at NLO. (ii) The potentials in the 2-nucleon sector are approximately equivalent.

\section{RESULTS FOR THREE AND FOUR NUCLEONS}

We now present the results of our feasibility study of using the RRGM with EFTs, exemplified at NLO calculations in $\mathrm{EFT}_{\not}$. The section is divided into four subsections. The first reports on an analysis of the correlation between the triton charge radius and its binding energy. The second looks at the splitting of the trinucleon binding energies due to Coulomb effects. The third discusses the Tjon correlation, and the fourth contains the findings for the 3-Helium-neutron scattering system.

\section{A. Three nucleons: triton charge radius}

Friar et al. [46] observed a correlation between the triton binding energy $B(t)$ and the triton charge radius using various nuclear force models, before Platter et al. [41] showed by a variation of the three-body interaction parameter at leading-order that it can be understood as a consequence of universality in $\mathrm{EFT}_{\not}$. With the model spaces defined in sect. III $B(t)$ and the corresponding ground state wave function $\langle\vec{r} \mid t\rangle$ were calculated at LO and NLO, and from that the root mean square charge radius $r_{\mathrm{ch}}^{t}$ of the triton:

$$
r_{\mathrm{ch}}^{t}=\left(\left\langle t\left|\sum_{i=1}^{3} \frac{1}{2} \vec{r}_{i}^{2}\left(1+\tau_{i}^{3}\right)\right| t\right\rangle\right)^{\frac{1}{2}},
$$

where $\vec{r}_{i}$ is the position and $\left(1+\tau_{i}^{3}\right)$ the charge operator of the $i$-th nucleon. The results in fig. 2 confirm the expected behavior of an increasing $r_{\mathrm{ch}}^{t}$ for more loosely bound systems. Results of two EFT LO calculations are shown. The RRGM LO potentials map out a band (gray shaded area) which includes the datum. The lower and upper LO line from [41] (dashed gray lines in fig. 2) result from a fit of the LO LECs $C_{1,2}^{\mathrm{LO}}$ to either $a_{s}, a_{s}$ or $B(d)$, $a_{s}$, respectively, providing some measure of higher order effects. For the RRGM LO calculation, we used $a_{s, t}$ to determine the LECs, and varied the cutoff from $200 \mathrm{MeV}$ (top edge) to $1.6 \mathrm{GeV}$ (lower edge) in steps of $100 \mathrm{MeV}$. We choose the lower bound for $\Lambda$ to be in the region of the pion mass. The upper bound is set by the observation that there is essentially no change for cutoffs larger than $1.6 \mathrm{GeV}$. The band appears to be saturated, with the bulk of its width coming from the region $\Lambda \in[400 ; 800] \mathrm{MeV}$. For fixed $\Lambda$, the 3NI was finally tuned to a given $B(t)$ to generate the correlation lines.

The quasi-exact Faddeev calculation of [41] and the RRGM results do not overlap. Since app. A demonstrates that numerical inaccuracies of the RRGM are negligible, we speculate that this can be traced to the differences between the regularization schemes employed. The Faddeev calculation uses the separable cutoff function $f_{\kappa}\left(\vec{p}, \vec{p}^{\prime}\right)=\exp \left(-\vec{p}^{2} / \kappa^{2}\right) \exp \left(-\vec{p}^{2} / \kappa^{2}\right)$ with $\kappa \geq 1600 \mathrm{MeV}$, while the RRGM uses the non-separable regulator $f_{\Lambda}\left(\vec{p}, \vec{p}^{\prime}\right)=\exp \left(-\left(\vec{p}-\vec{p}^{\prime}\right)^{2} / \Lambda^{2}\right)$, plus an implicit regulator imposed by the finite number of width parameters. Therefore, both methods combined can be viewed as providing a check of residual regularization-scheme dependence. A conservative estimate of LO effects is thus the range of results coated by the combination of both methods. The LO accuracy at the physical triton binding energy is thus $\pm 0.6 \mathrm{fm}$, and the measured charge radius happens to lie right in the middle of the LO band. Additional regularization schemes, e.g., with a range of cutoff values in the Faddeev approach, are thus conjectured to lead to an overlap between the results of [41] and the RRGM bands. 


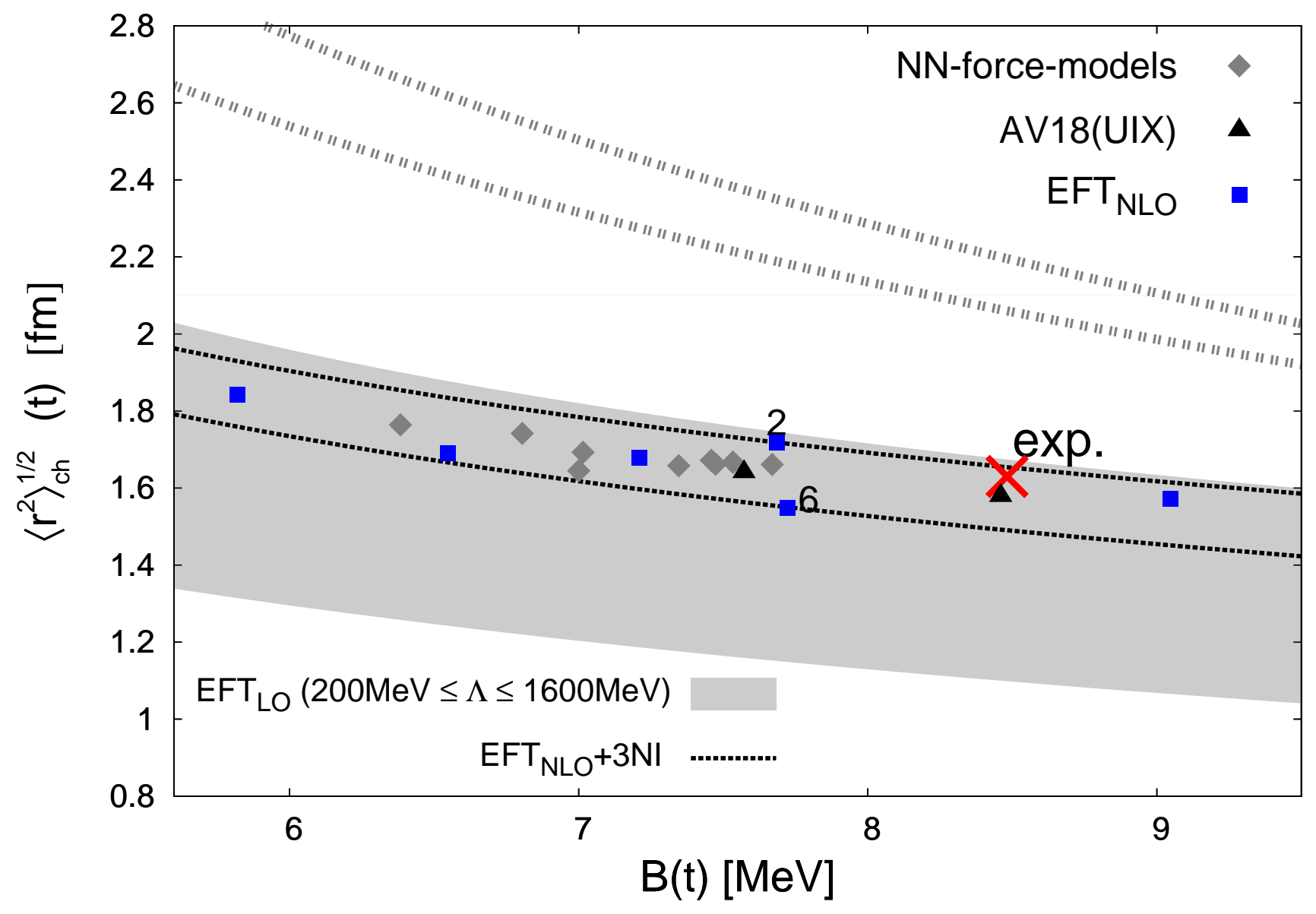

FIG. 2: The correlation between the triton charge radius and its binding energy. The narrower band mapped out by the $\mathrm{EFT}_{\not} \mathrm{NLO}$ results from the RRGM (blue squares, black dashed lines) as described in the text, compared to our RRGM LO results (gray shaded area) and a LO calculation by [41] (dashed gray lines), demonstrates convergence, also to the datum ( $r_{\text {ch }}^{t}$ from [42], $B^{\exp }(t)$ from [43]). The values from AV18(+UIX) (black triangles, RRGM calculation for this work) and a variety

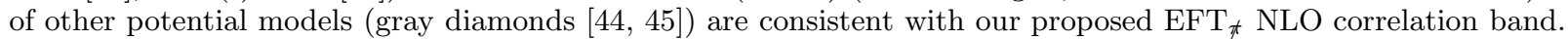

At NLO, the potentials without 3NI map out a correlation band which is more narrow than its LO counterpart, and again contains the datum. The width of the band can be estimated by considering the difference between the results using the extreme cases provided by potentials ${ }^{2} V_{\text {t }}$ and ${ }^{6} V_{\text {t }}$. They produce the same NN scattering lengths and nearly the same triton binding energy, and their deuteron binding energies differ by less than $10 \%$, while their triton charge radii differ by about $10 \%$, consistent with the expectation of a NLO calculation. Finally, they are based on two significantly different cutoff values, $\Lambda\left({ }^{2} V_{\star}\right) \approx 400 \mathrm{MeV}$ and $\Lambda\left({ }^{6} V_{\star}\right) \approx 650 \mathrm{MeV}$. We therefore can base an estimate of the NLO band on the range mapped out by varying the $3 \mathrm{NI}$ for these two potentials. These lines are included in fig. 2,

Variation of the cutoff, of the fitting-input, and of the 3NI lead therefore all to similar assessments of the uncertainty of the theory at NLO. At fixed $B(t)$, the charge radius varies from LO to NLO by $\lesssim 30 \%$, in agreement with the power-counting which estimates the correction from order to order to scale as $Q \sim p_{\text {typ }} / \Lambda_{b} \approx \frac{1}{3}$.

All three values, namely, $\pm 0.2 \mathrm{fm}$ from the naïve estimate $Q \approx \frac{1}{3}$ and the observed convergence from LO to NLO, and $\pm 0.1 \mathrm{fm}$ from the above mentioned difference between ${ }^{2} V_{\star \star}$ and ${ }^{6} V_{\star t}$, would be equally valid estimates for the theoretical uncertainty of this NLO calculation. As demonstrated in app. A errors induced by numerical inaccuracies are negligible. Using the more conservative error estimate and the assumption for the NLO error band center at $1.6 \mathrm{fm}$ at the experimental $B(t), \mathrm{EFT}_{\text {力 }}$ predicts a value

$$
r_{c h}^{t, \mathrm{NLO}}=(1.6 \pm 0.2) \mathrm{fm}
$$

within the band of the leading-order value $r_{c h}^{t, \mathrm{LO}}=(2.1 \pm 0.6) \mathrm{fm}$ as quoted from [41]. The NLO value is found in 
good agreement with experiment [42], $r_{c h}^{\exp }=(1.63 \pm 0.03) \mathrm{fm}$.

Another argument in favor of our definition of the correlation band is provided by the results of the two phenomenological models, AV18 35] and AV18+UIX [47]. In general, EFT predicts that the results of a potential which reproduces or shares input observables at least to the accuracy required at the considered order, deviate from the results of an appropriate EFT potential by less than the theoretical uncertainty of the EFT values at this order in the applicability range of the EFT. This criterion is easily met by AV18(+UIX), and hence its predictions have to be consistent with the proposed correlation band. The two-body potential AV18, reproducing the Nijmegen phases much more accurately than required to fall into this category of potentials, is expected to yield a value at a position within the $\left(r_{c h}^{t}-B(t)\right)$-band. The prediction for the triton charge radius of AV18+UIX, however, is expected to deviate less than $10 \%$ from the experimental datum within the error band, because this model has a three-body interaction added to reproduce the experimental $B(t)$. Both expectations are consistent with the results shown in fig. 2

In conclusion, the results for these 3-nucleon observables show that although the potentials are approximately NN phase shift equivalent, they differ in their predictions of three-body observables. As mentioned in the introduction, a proper renormalization of the theory requires therefore one 3 -nucleon contact interaction. Setting this $3 \mathrm{NI}$ to zero in the potentials ${ }^{1-9} V_{\mathbb{t}}$, the expected dependency of observables in $A>2$ systems on how the unobservable short-distance physics is modeled is observed. Different short-distance physics is modeled by the potentials not only by varying cutoff values but also by differing sets of LECs for the same cutoff, while the scheme- and regulator-dependent three-body interaction parameter is chosen as zero. With this parameter fitted to the triton binding energy, the prediction for the triton charge radius is consistent with experiment within the expected uncertainty range. A significant convergence from LO to NLO is observed.

\section{B. Three nucleons: effect of the Coulomb force}

In fig. 3, the RRGM results for the splitting between the binding energies of 3-Helium and the triton are shown for the potentials ${ }^{1-9} V_{\not \nmid}$. At NLO in the pion-less EFT, the strong interaction is isoscalar and hence does not break charge symmetry. Therefore, charge symmetry breaking (CSB) comes in our RRGM calculation only from including the Coulomb interaction between the protons in ${ }^{3} \mathrm{He}$. The $\mathrm{EFT}_{\star}$ results, hence, only show the model independent contribution of Coulomb interactions to the trinucleon binding energy splitting. The correlation band is mapped out by the $\mathrm{EFT}_{\not \text { }}$ potentials with zero $3 \mathrm{NI}$ and by smoothly varying this three-body parameter for potentials ${ }^{1,7} V_{\not \nless}$. Both approaches result in correlations consistent with each other and with the results of the other NLO potentials. At the experimental triton binding energy, this leads to predicting

$$
\left(B(t)-B\left({ }^{3} \mathrm{He}\right)\right)=(0.66 \pm 0.03) \mathrm{MeV}
$$

Here, we estimate the theoretical uncertainty by the spread of the phase-equivalent NN potentials as in the previous section, e.g. comparing ${ }^{3,9} V_{\not}$. The a priori error estimate at $\mathrm{NLO}$ of $\lesssim 10 \%$ gives a larger uncertainty of $\pm 0.07 \mathrm{MeV}$. However, one should keep in mind that including iso-scalar strong interactions by higher order terms of the effectiverange expansion has identical effects on the strong interactions inside the triton and ${ }^{3}$ He. These effects cancel out in the difference and only survive indirectly, as the strength of the Coulomb interaction in a system is also correlated to its size. We therefore quote the width of the correlation band as error estimate of our calculation.

This value deviates by about $0.1 \mathrm{MeV}$ from the experimental value of $0.764 \mathrm{MeV}$, see e.g. the recent review on CSB and Charge Independence Breaking (CIB) [50]. In line with the argumentation above, we attribute this difference to isospin breaking CIB/CSB interactions coming from the explicitly broken chiral symmetry in the strong and electroweak sector from which only the parts resulting in the Coulomb force have been considered in this calculation. They enter in $\mathrm{EFT}_{\not \text { 木 }}$ only at higher order. To support this assertion, results of the potential models AV18(+UIX), which contain CSB interactions, are included in fig. 3. Both potentials are not elements of the correlation band suggested by the $\mathrm{EFT}_{\not \nless}$ points but agree with a shifted band, centered around the datum. In contrast, the values from charge symmetric potential models in fig. 3 lie within the NLO EFT $\mathrm{CSB} / \mathrm{CIB}$ contribution to the binding difference in NLO EFT $\mathrm{N}_{\not}$ at the experimental triton binding energy of

$$
\left(B(t)-B\left({ }^{3} \mathrm{He}\right)\right)^{\mathrm{CSB} / \mathrm{CIB}}=(0.10 \mp 0.03) \mathrm{MeV}
$$

anti-correlated with the Coulomb contribution to give the experimentally established difference. This is to be compared with the contributions from 2- and 3-nucleon CSB interactions which stem from Chiral Effective Field Theory, Breit and vacuum polarization corrections, and from corrections to the kinetic energy operator. In Ref. [49], these were calculated to sum up to $(0.112 \mp 0.022) \mathrm{MeV}$, leaving about $(0.652 \pm 0.022) \mathrm{MeV}$ for the soft photon effects, dominated by the Coulomb interaction. This is in perfect agreement with the $\mathrm{EFT}_{\not}$ result. 


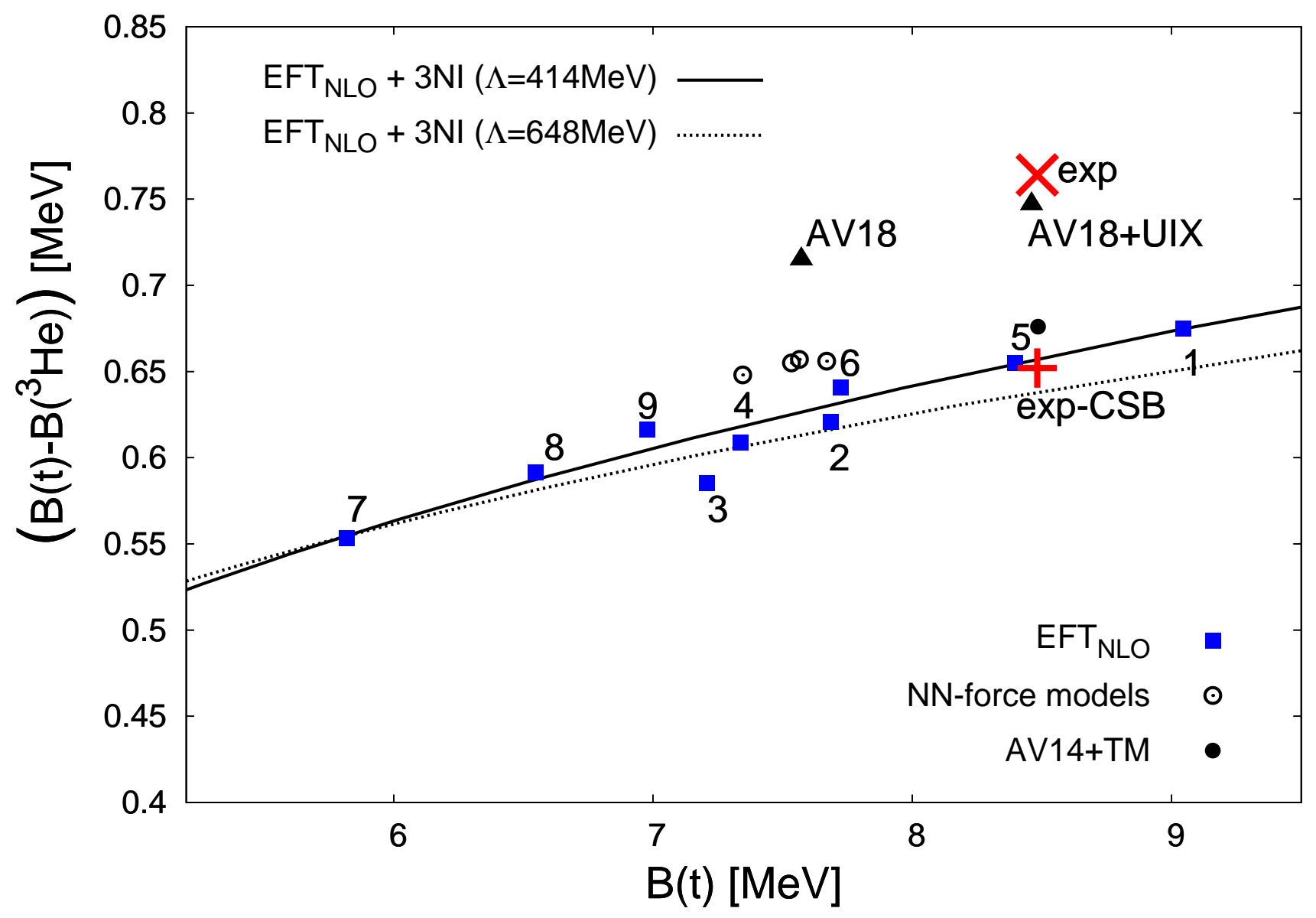

FIG. 3: Binding energy difference between ${ }^{3} \mathrm{He}$ and ${ }^{3} \mathrm{H}$ for $\mathrm{EFT}_{\not \nless}$ potentials (filled squares) compared to various isospin invariant potential models calculations [45, 48] (circles) and RRGM values for AV18(+UIX) (triangles) which contain charge symmetry breaking terms. For the potentials ${ }^{1} V_{\star}$ (solid) and ${ }^{7} V_{\star}$ (dashed), a smooth variation of the 3 NI leads to the two correlation lines. The upright cross is the experimental value without the contribution from CSB terms as found in [49].

\section{Four nucleons: bound state}

Venturing into the four-body system, we now present results of the Tjon correlation line 12] including Coulomb interactions between the ground state energies of the triton and 4-Helium at LO and NLO, extending the LO analysis of [10], where the effect of Coulomb interactions was only estimated.

Before reporting the results, we describe the employed variational space and its construction. The model space in which the 4-Helium binding energy $B(\alpha)$ was initially calculated is spanned by vectors defined in eq. (3) with relative angular momenta $l_{k i} \leq 2$ including all coupling schemes to yield a total angular momentum state $J^{\pi}=0^{+}$and width parameters $\gamma_{d k}$ to allow for the formation of triton, 3-Helium, and deuteron fragments. As mentioned above, the $w_{63}$ sets were used for the deuteron, resulting in 169 configurations. For the 3-nucleon fragments, a small model space of dimension $d \leq 70$ was generated for each potential as follows. To an initial set of 20 basis vectors which bind the triton, one new basis vector was added, and its two width parameters $\gamma_{d k}$ (see eq. (3D) optimized with the genetic algorithm to maximize the gain in binding energy. This process was iterated until $\left|B^{224}(t)-B^{\text {small }}(t)\right| \leq 500 \mathrm{keV}$, where the superscript labels the aforementioned 224-dimensional model space. For 3-Helium, a copy of this model space was used which differed only in its isospin quantum numbers. On average, 300 configurations were considered for the model space of a given $V_{\star}$, for each of which four or five inter-fragment width parameters were used, resulting in roughly 1200 basis vectors.

To assess if the reduction in the number of triton components still guaranteed for an almost complete 4-Helium model space, the binding energy $B(\alpha)$ thus obtained was compared to one calculated in the much larger scattering model space. Nevertheless, the two values differed by less than $50 \mathrm{keV}$. This model space, used for the $0^{+}-$channel 


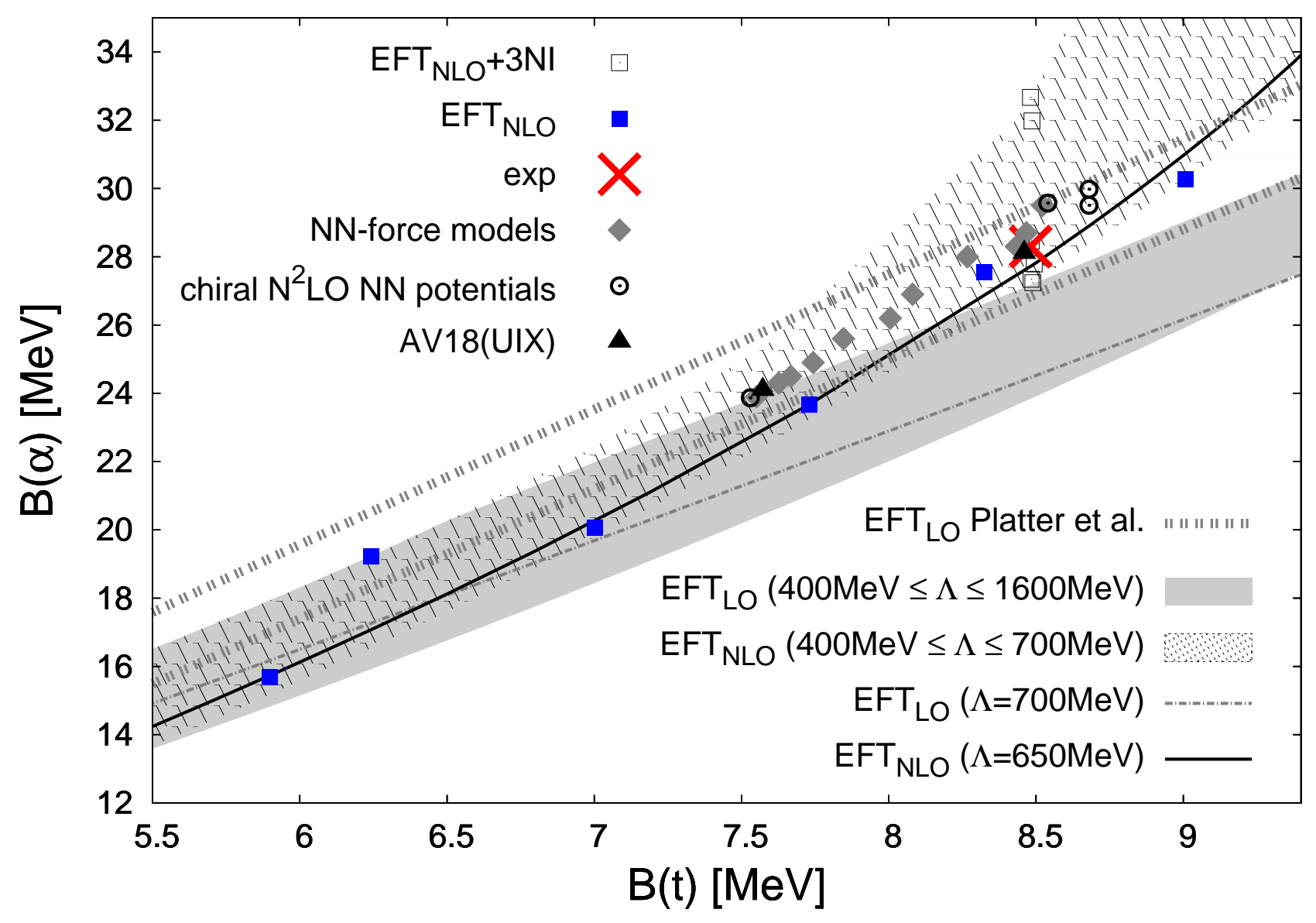

FIG. 4: Correlation between the triton and ${ }^{4} \mathrm{He}$ binding energies (Tjon line). The filled squares are the results using NLO $\mathrm{EFT}_{\not}$ NN potentials from table【with no $3 \mathrm{NI}$, and the empty squares represent the predictions for the $3 \mathrm{NI}$ fitted to $B(t)$. The shaded NLO band results from a variation of the 3NI for those potentials. We compare to LO results from [10], where the upper (lower) dashed line was calculated with LEC fitted to $a_{s, t}\left(B(d), a_{s}\right)$, and to our RRGM calculation for $\Lambda$ ranging from $400 \mathrm{MeV}$ (lower edge) to $1.6 \mathrm{GeV}$ (top edge of light gray area). $B^{\exp }(\alpha)$ is taken from [51], the values for AV18 $(+\mathrm{UIX})$ are reported in [52], and those using a chiral expansion to NLO and $\mathrm{N}^{2} \mathrm{LO}$ in [7].

scattering calculation is defined below and is spanned by more than 7000 basis vectors. This study of reducing the dimension of the 3-nucleon fragments in the $\alpha$-particle without deviating significantly from the assumed converged value is crucial for future applications of the method to $A>4$ systems.

In fig. 4 the results are compared to the LO band calculated by Platter et al. 10]. The spread of the NLO values is not in conflict with $\mathrm{EFT}_{\not}$ which allows a $\lesssim 10 \%$ uncertainty at NLO. As for the triton charge radius, Platter et $a l$. obtained the upper (lower) boundary of the LO band by choosing different NN observables, $a_{s, t}\left(B(d), a_{s}\right)$, to fit the LO LECs for cutoffs high enough so that $B(\alpha)$ did not change when further changing the cutoff. They already pointed out that this gives only a crude estimate of higher order effects. Figures 2 and 4 confirm that the LO accuracy would be overestimated with this method. Our LO calculation uses the same interactions as in sect. VA, with $a_{s, t}$ as input, and cutoff values from $400 \mathrm{MeV}$ (lower bound of $\mathrm{LO}$ band in fig. (4) to $1.6 \mathrm{GeV}$ (upper bound). In contrast to our RRGM LO correlation between $B(t)$ and the triton charge radius, the width of the RRGM LO Tjon band is however not converged. The shifts between the positions of the correlation lines corresponding to cutoffs of $400 \mathrm{MeV}$ (lower edge of gray area in fig. 4), $700 \mathrm{MeV}$ (dashed dotted line), and $1.6 \mathrm{GeV}$ indicate that a variation of $\Lambda$ even beyond $1.6 \mathrm{GeV}$ would be necessary to assess the LO uncertainty from cutoff variations only. However, elaborate technical modifications are required for the RRGM calculation at higher cutoffs. Analogous to the conservative estimate for the LO uncertainty in fig. 2, the LO Tjon correlation band is thus mapped out by both the RRGM and the Faddeev/Yakubovsky (thick dashed lines) results, which overlap nicely in this plot. This combined correlation band includes the datum and the narrower NLO band, which, from fig. [4 has at the experimental $B(t)$ a width of 
about $5 \mathrm{MeV}$ centered around $28 \mathrm{MeV}$ and results in a prediction of

$$
B^{\mathrm{NLO}}(\alpha)=(28 \pm 2.5) \mathrm{MeV}
$$

which is consistent with the expected NLO uncertainty of about $10 \%$ and with experiment. Again, the results of the AV18(+UIX) models lie within the proposed band as it is expected of all interaction models of at least NLO. The observed broadening of the correlation band is a manifestation of the momentum dependence of the EFT expansion. The accuracy decreases with increasing typical momentum, eventually leading to a breakdown of the expansion.

From the fact that there is still a one-parameter correlation, we conclude that no four-nucleon contact interaction is required to renormalize the theory at NLO. One three-body parameter fitted to data suffices to yield proper NLO predictions for four-body observables within the theoretical accuracy (empty squares in fig. 4).

\section{Four nucleons: scattering}

We now turn to scattering observables. In principle, all low-energy observables should be correlated with the triton binding energy. The recent results [53] for the singlet and triplet $n-{ }^{3} \mathrm{H}$ scattering lengths using three potential models and a $\mathrm{N}^{3} \mathrm{LO}$ chiral potential are evidence for this assertion in the four-nucleon scattering system. Here, the real part of the S-wave spin singlet scattering length $a_{0}\left({ }^{3} \mathrm{He}-\mathrm{n}\right)$ for elastic 3 -Helium-neutron scattering is investigated. In fig. 5 , its value is shown as a function of $B(t)$ for six potentials $V_{\not t}$ including the Coulomb interaction. For two NLO potentials with $\Lambda=440 \mathrm{MeV}$ (solid line) and $\Lambda=550 \mathrm{MeV}$ (dashed line), with 2-nucleon LECs fixed, we also show the effect of a smooth variation of the 3 -nucleon interaction parameter.

To extract $a_{0}\left({ }^{3} \mathrm{He}-\mathrm{n}\right)$, six two-fragment channels, ${ }^{3} \mathrm{He}-\mathrm{n}, \mathrm{t}-\mathrm{p}, \mathrm{d}-\mathrm{d}\left(l_{\mathrm{rel}}=0,2\right)$, (nn)-(pp), and dq-dq (dq is the singlet "deuteron" with $S=0$ ), are included. The latter two consist of unbound fragments and usually model possible threeand four-body breakup reactions. They, as well as the two d-d channels, are however for this calculation only needed to provide configurations for distortion channels since only the t-p channel is open a few eV above the ${ }^{3} \mathrm{He}-\mathrm{n}$ threshold. For the fragment wave functions $\psi_{j}$ (see eq. (4) ), a 224 dimensional basis was used for the triton and 3-Helium, and a 9 dimensional one for the deuteron, whose six $L_{j}=0$ vectors built the nn, pp, and dq states. For these six channels, the 20 width parameters $w_{12}$ were used for the $\omega_{j m}$ in eq. (4). Almost all configurations included to build those physical channels could be recycled as distortion channels to allow for more freedom in the minimization of the variational functional. Less than ten configurations had to be excluded to avoid numerical linear dependences. In each distortion channel, four to six relative width parameters $\omega_{j m}$, taken from $w_{12}$ with $\omega_{j m}>0.02$ fm $^{-2}$, were used. Numerical stability and convergence of $a_{0}\left({ }^{3} \mathrm{He}-\mathrm{n}\right)$ were assessed by increasing the number of included relative widths $\omega_{j m}$ by one for each distortion channel, yielding changes in $a_{0}\left({ }^{3} \mathrm{He}-\mathrm{n}\right)$ of the order of the numerical uncertainties, given that the initial $\omega_{j m}$ were chosen appropriately. The lowest eigenvalue of the Hamiltonian is equal to the ground state energy of 4-Helium in this model space and was allowed to change in this process by not more than $10 \mathrm{keV}$. Significantly larger changes in this eigenvalue which leads to a result not of the order of magnitude suggested by the LO Tjon band signal numerical linear dependences. If the model space is too small, or if the width parameters for the relative wave function were chosen inappropriately, changes of the order of $100 \mathrm{keV}$ up to a few $\mathrm{MeV}$ are expected.

The 3-Helium-neutron scattering length $a_{0}\left({ }^{3} \mathrm{He}-\mathrm{n}\right)$ was calculated from the corresponding diagonal element $S_{22}$ of the S-matrix at a center of mass energy of $E_{\mathrm{cm}}<10 \mathrm{eV}$ above the ${ }^{3} \mathrm{He}-\mathrm{n}$ threshold analogously to [54]:

$$
a_{0}\left(E_{\mathrm{cm}}\right)=\frac{1-S_{22}\left(E_{\mathrm{cm}}\right)}{i \sqrt{\frac{3}{4} M E_{\mathrm{cm}}}\left(1+S_{22}\left(E_{\mathrm{cm}}\right)\right)} .
$$

A fraction of the flux is diverted into the open triton-proton channel, resulting in a nonzero $S_{12}$ S-matrix element and hence a nonzero imaginary part of $a_{0}\left({ }^{3} \mathrm{He}-\mathrm{n}\right)$ which is not reported here. The $3 \mathrm{NI}$ variation yields an almost linearly increasing imaginary part with increasing $B(t)$ from about $\operatorname{Im}\left\{a_{0}\left({ }^{3} \mathrm{He}-\mathrm{n}\right)\right\} \approx-7.5 \mathrm{fm}$ at $B(t) \approx 5.5 \mathrm{MeV}$ to $\operatorname{Im}\left\{a_{0}\left({ }^{3} \mathrm{He}-\mathrm{n}\right)\right\} \approx-2.0 \mathrm{fm}$ at $B(t) \approx 9.1 \mathrm{MeV}$. This qualitative observation is consistent with the decreasing trinucleon binding energy splitting with decreasing triton binding energy (see sect. VB), which results in a smaller separation between the respective thresholds in four-nucleon scattering. This handle on the threshold separation suggests an approach to circumvent numerical problems associated with the proximity of thresholds by extrapolating results for their physical values from calculations performed at more deeply bound 3-nucleon states.

In fig. 5] the predictions of the NLO potentials with and without $3 \mathrm{NI}$ for $\operatorname{Re}\left\{a_{0}\left({ }^{3} \mathrm{He}-\mathrm{n}\right)\right\}$ decrease with increasing triton binding energy. They map out a band which includes the datum. A comparison to hard sphere scattering qualitatively explains this behavior. A higher 3 -Helium binding energy $B\left({ }^{3} \mathrm{He}\right)$ corresponds to a smaller nucleus analogous to the triton as shown in fig. 2. As the scattering length is proportional to the radius of the hard sphere, $a_{0}\left({ }^{3} \mathrm{He}-\mathrm{n}\right)$ is expected to decrease for increasing $B\left({ }^{3} \mathrm{He}\right)$. We define the NLO correlation band to be centered around 


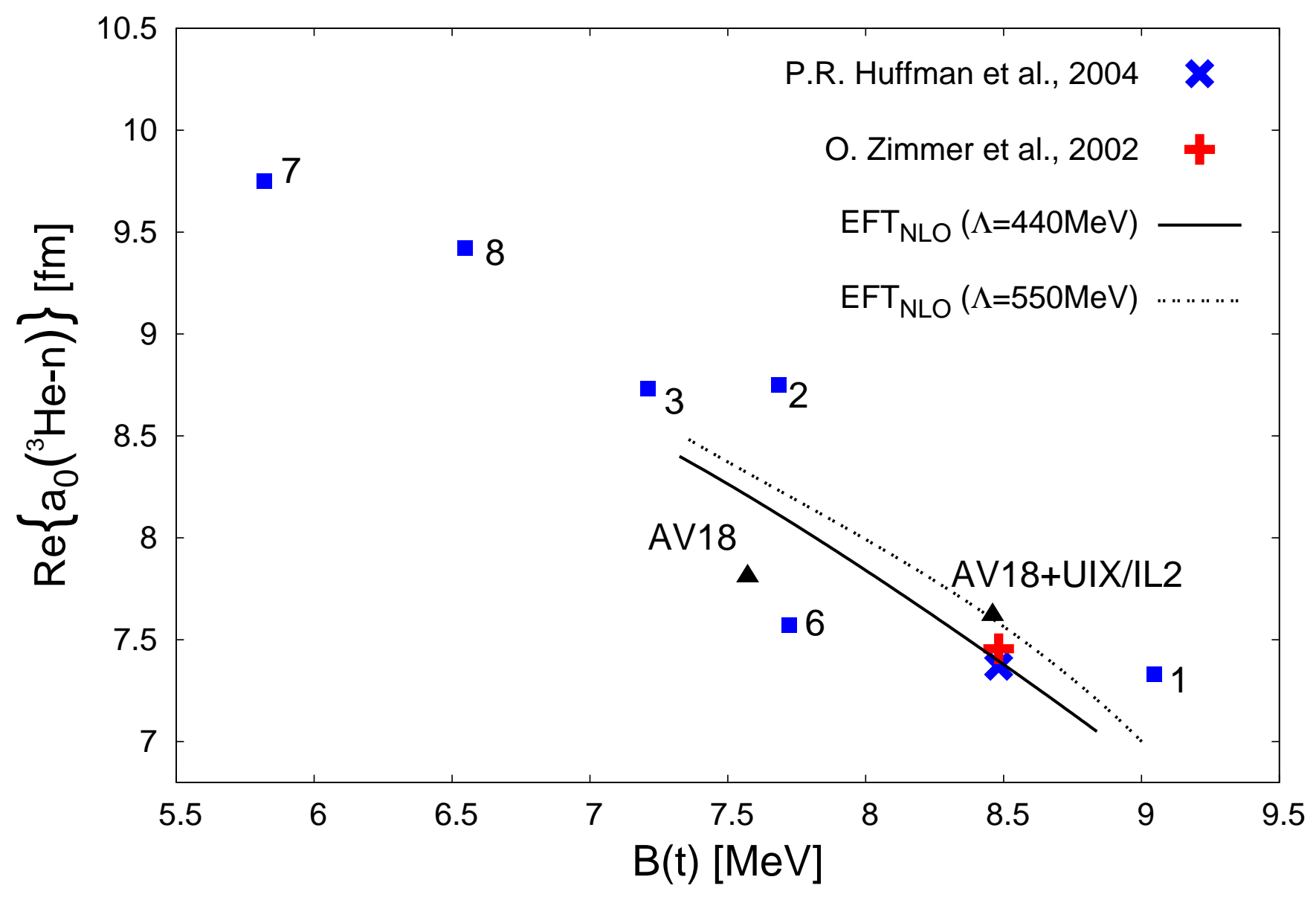

FIG. 5: The correlation between the triton binding energy and the real part of the spin singlet, S-wave scattering length $a_{0}\left({ }^{3} \mathrm{He}-\mathrm{n}\right)$ for elastic ${ }^{3} \mathrm{He}-\mathrm{n}$ scattering. The dots represent the values of the NLO EFT $\mathrm{E}_{\star}$ potentials with a numerical uncertainty of $\mathcal{O}(0.02 \mathrm{fm})$. A variation of the 3NI yields the continuous correlation lines for two selected NLO potentials with a fixed cutoff.

the values predicted by the potentials with zero 3 NI. The change in $a_{0}\left({ }^{3} \mathrm{He}-\mathrm{n}\right)$ observed between ${ }^{2} V_{\star}$ and ${ }^{6} V_{\star}$ serves as an error estimate for the band centered around $7.5 \mathrm{fm}$. Again, the assumption of the center of the band at the experimental $B(t)$ follows from the values in fig. 5 . Thus, EFT , N $^{\mathrm{NLO}}$ reports

$$
\operatorname{Re}\left\{a_{0}\left({ }^{3} \mathrm{He}-\mathrm{n}\right)\right\}=(7.5 \pm 0.6) \mathrm{fm} .
$$

The 3NI was varied only over the depicted range of trition binding energies, where one and the same fixed RRGM variational space can be used. The fragment model space for the two interactions was optimized for the 3NI parameter fitted to $B(t)$. Only the number of included distortion channels was changed to reach convergence within this space for each value of the $3 \mathrm{NI}$. The band mapped out by the two $3 \mathrm{NI}$ lines for $(7.5 \mathrm{MeV} \lesssim B(t) \lesssim 9 \mathrm{MeV})$ includes the datum, and its slope is consistent with the one indicated by the 6 NLO potentials without 3NI. This explicitly demonstrates that a variation of the $3 \mathrm{NI}$ has the same effect as varying the short-distance part of the NN interaction. The NN model AV18 yields a value within the error band. Adding the UIX three-body interaction moves this point into the $10 \%$ NLO uncertainty radius around the datum. The numerical accuracy of this scattering calculation is assessed in app. B to be better than $1 \%$.

The conclusion drawn from the newly found correlation in fig. [5 is that every potential with the correct NN lowenergy phase shifts and appropriately tuned three-body interaction, e.g., to give the correct triton binding energy, predicts not only the correct $B(\alpha)$ but also the experimental $a_{0}\left({ }^{3} \mathrm{He}-\mathrm{n}\right)$ within a NLO error range. In table 【II, the results for $a_{0}\left({ }^{3} \mathrm{He}-\mathrm{n}\right)$ of RRGM calculations with the EFT without the three-body interaction models Urbana9 (UIX) and Illinois2 (IL2) [55] are given.

The values for the recent IL2 3NI have been calculated for this work, employing methods described in [52, 54]. The IL2 prediction for $\operatorname{Re}\left\{a_{0}\left({ }^{3} \mathrm{He}-\mathrm{n}\right)\right\}$ is almost identical to the UIX value and not plotted separately in fig. 坷, UIX and 
TABLE III: RRGM predictions for the triton binding energy and the spin singlet, S-wave scattering length $a_{0}\left({ }^{3} \mathrm{He}-\mathrm{n}\right)$ for elastic ${ }^{3}$ He-n scattering of phenomenological nucleon potential models. The values for AV18 and AV18+UIX are taken from [54], while the AV18+IL2 numbers are new results of this work. The imaginary part was investigated on a qualitative level, only.

\begin{tabular}{|c|c|c|c|}
\hline force & $B(t)[\mathrm{MeV}]$ & $\operatorname{Re}\left\{a_{0}\right\}[\mathrm{fm}]$ & $\operatorname{Im}\left\{a_{0}\right\}[\mathrm{fm}]$ \\
\hline $\mathrm{EFT}_{\sharp}^{\mathrm{NLO}}$ & 8.48 (input) & $7.5(6)$ & $-2.6(?)$ \\
\hline AV18 & 7.57 & 7.81 & -4.96 \\
\hline AV18+UIX & 8.43 & 7.62 & -4.07 \\
\hline AV18+IL2 & 8.48 & 7.63 & -4.28 \\
\hline \multirow{2}{*}{$\exp$} & \multirow{2}{*}{8.48} & $7.456(20)^{56}$ & \\
\hline & & $7.370(58)^{[57]}$ & \\
\hline
\end{tabular}

IL2 have parameters fitted to, amongst others, the triton and 4-Helium binding energies. The observed deviation of the prediction of both models for $a_{0}\left({ }^{3} \mathrm{He}-\mathrm{n}\right)$ is therefore not a result of a deficiency of the structure of the potential. The correlation between $B(t)$ and $a_{0}\left({ }^{3} \mathrm{He}-\mathrm{n}\right)$ supports the conjecture that this small deviation can only be improved by the inclusion of higher order interactions.

Analogously to UIX, the IL2 model easily satisfies the criteria mentioned in sect. VA namely to reproduce lowenergy NN observables and the triton binding energy at least with a $10 \%$ accuracy, and therefore should with its prediction for $a_{0}\left({ }^{3} \mathrm{He}-\mathrm{n}\right)$ also lie within a $10 \%$ radius of the datum in fig. 5. This EFT prediction is confirmed here by explicit calculation.

Both experimental values for $a_{0}\left({ }^{3} \mathrm{He}-\mathrm{n}\right)$ are included in the predicted universality band, and therefore we cannot resolve the discrepancy between the two measurements.

\section{E. Evidence for the absence of four-nucleon interactions at NLO}

We close with a comment on the implications of these results for the scaling of four-nucleon interactions (4NI) in $\mathrm{EFT}_{\text {\# }}$ at LO and NLO.

Simplistic dimensional analysis suggests that a momentum-independent $4 \mathrm{NI}$ enters at $\mathrm{N}^{4} \mathrm{LO}$, but the unusual renormalization of the $3 \mathrm{NI}$ may also promote the $4 \mathrm{NI}$ to contribute at lower orders to ensure renormalizability. By varying their harsh cutoff between $800 \mathrm{MeV}$ and $2000 \mathrm{MeV}$, Platter et al. [10] concluded that a 4NI is not necessary at $\mathrm{LO}$ for cutoff-independence of the 4-Helium binding energy.

In the present approach with a Gaussian regulator, the cutoff was varied for the 4-Helium binding energy and for the 3-Helium-n scattering length from $400 \mathrm{MeV}$ to $700 \mathrm{MeV}$. This led to a correlation band near-identical with the ones mapped out when using different NN potentials (in part also at different cutoffs) or different 3NIs. All results were converged numerically. Moreover, we demonstrated that the theoretical uncertainty for $B(\alpha)$ decreases from LO to NLO by a factor consistent with the a-priori expansion parameter estimate $Q \approx \frac{1}{3}$. In both cases, the physical datum lies well inside the NLO correlation band.

Let us assume now that a 4NI enters at NLO. Our not accounting for it would then have two effects: The results could be unstable against cutoff variations, indicating that a $4 \mathrm{NI}$ is necessary at NLO to renormalize EFT . We see no such effect, but are aware that the cutoff might have to be varied beyond the window chosen in this exploratory study. Secondly, the theoretical accuracy of the results would be reduced to LO, even if results independent of the cutoff can be achieved, i.e., even if the cutoff can be removed to infinity without a 4NI. In other words, when one separately or combinedly varies the cutoff or the 2 -nucleon potential used or the $3 \mathrm{NI}$ strength, the different results should spread in a corridor set by the size of LO corrections, i.e., $\lesssim 30 \%$, and not by the corridor of $\lesssim 10 \%$ expected in a NLO calculation. We have demonstrated above for both observables that the residual short-distance dependence of the observables is $\lesssim 10 \%$ of the central value, taking as conservative estimate the combination of errors which occur when varying $\mathrm{EFT}_{\not \text { t }}$ at unphysically short distances: Different, phase equivalent NN potentials; different 3NIs; different cutoffs. The correlation bands and error estimates are thus on the quantitative level consistent with those of a NLO calculation.

We also note in passing that the physical datum lies well inside the NLO correlation band for both four-nucleon observables. A residual 4NI not necessary for renormalization but with unnaturally large coefficient can therefore be ruled out.

Our conclusion therefore is: There is strong evidence that 4 NIs do not enter at either LO or NLO in EFT . An indisputable criterion to assess whether our interpretation is correct can be provided in a future study [58] of the convergence pattern of the momentum-dependence of four-nucleon observables from zero to the breakdown scale. In it, an observable calculated up to order $Q^{n}$ must show a residual short-distance dependence on compatible with $n$ 
powers of the typical low-energy momentum. This analysis will not re-sum the effective range contributions into the 2-nucleon propagator, but treat higher order effects in strict perturbation.

\section{CONCLUSIONS}

The effective field theory formalism can be used to explain empirically found correlations amongst few-nucleon observables like the Phillips- and Tjon line. In these two cases, it relates the deviations from data to, first, an incomplete renormalization in the 3-nucleon sector, and second, to higher order interactions omitted in the course of the EFT expansion. The theoretical uncertainty at every order of the calculation can be quantified in this approach. Numerical inaccuracies were demonstrated to be negligible in app. A and app. B. Different results for different shortdistance parameterizations therefore have other origins. The size of the expansion parameter $p_{\text {typ }} / \Lambda_{b}$ determines how fast an EFT expansion converges and decides its usefulness for the calculation of an observable in a given system. In heavier systems like 4-Helium, the expansion parameter can a priori be as large as 1 , so they are border line. However, we find that next-to-leading order corrections to leading-order results are still parametrically small in the four-nucleon system. This confirms a pattern already seen in 2- and 3-nucleon systems at momenta which approach the a priori breakdown scale but where convergence is still found, see e.g. [9, 22, 27, 28].

The computational challenges of calculating few-nucleon observables are met in this work by the Refined Resonating Group Method. It provides a versatile method for bound- and scattering properties. Here, it was found highly economical with respect to computer time when combined with the $\mathrm{EFT}_{\not \star} \mathrm{NN}$ potentials derived in sect. IV] for a range of momentum cutoff values and with a full treatment of the Coulomb interaction.

For two correlations for which leading-order calculations exist, namely between the binding energy and charge radius of the triton in sect. $\overline{\nabla A}$ and the binding energies of 4-Helium and the triton in sect. $\nabla \mathrm{C}$, our coordinate space $\mathrm{EFT}_{\not}$ calculations at NLO report the expected improvement from LO to NLO consistent with an expansion parameter

$p_{\text {typ }} / \Lambda_{b} \approx \frac{1}{3}$. By that we demonstrated that a consistent description of the $\alpha$-particle is possible at NLO in EFT $\mathrm{T}_{\not h}$ In sect. $\mathrm{VB}$, we also report a correlation between the triton binding energy and its difference to the ${ }^{3}$ He binding energy. As the $\mathrm{EFT}_{\not}$ potential is at NLO iso-spin symmetric, this model independent difference is attributed to Coulomb interactions only, which are included in the RRGM. At the physical triton binding energy, this value agrees well both in magnitude and uncertainty with estimates of charge-symmetry breaking and Coulomb contributions to ${ }^{3} \mathrm{He}$ binding. In sect. VD, a new correlation between the triton binding energy and the real part of the singlet S-wave scattering length of 3-Helium-neutron scattering similar to the Tjon line is also found. This, and the three aforementioned correlation bands, let us also conclude that no four-body contact interaction is required to renormalize the system at next-to-leading order. The position of all four bands, which represent universal properties of the 2-nucleon system, was determined by fitting nine NN potentials differing at short-distances but with identical long-distance behavior, by variations of the $3 \mathrm{NI}$ strengths, and by changing the cutoff.

Consistent with a basic tenet of EFT, namely model independence, we also showed that the results of the phenomenological models AV18(+UIX/IL2), which share the input of our EFT $\mathrm{ELO}_{\not}$ NLO potentials, agree with their results within NLO accuracy.

Future work will utilize the relatively fast computations of four-nucleon observables, which result from the harmonic interplay of the RRGM with EFT $\mathrm{E}^{\mathrm{NLO}}$ potentials, in analyses of universal properties of $A>4$ systems. The Borromean halo nucleus, 6-Helium, is of special interest because $\mathrm{EFT}_{\not}$ calculations in this system can also provide input for $\alpha N$ effective field theories [59]. Furthermore, we are now equipped for a study of electro-weak interactions with heavier nuclei like ${ }^{4} \mathrm{He}(\gamma, \mathrm{p})$ and ${ }^{4} \mathrm{He}(\gamma, \mathrm{n})$ using $\mathrm{EFT}_{\not \nless}$ and the RRGM, to address both conflicting measurements as well as theoretical calculations [60 62]. Valuable input for astrophysical calculations for the prediction of light element abundances can also be provided by a calculation of reaction cross-sections for e.g. $\mathrm{d}(\mathrm{d}, \mathrm{n})^{3} \mathrm{He}$ and $\mathrm{d}(\mathrm{d}, \mathrm{p}) \mathrm{t}$ at very low energies.

\section{Acknowledgments}

We thank M.C. Birse, G. Hager, H.W. Hammer, C. Pelissier, D.R. Phillips, and G. Wellein for helpful discussions. JK acknowledges the kind hospitality of the Dipartimento di Fisica of the Università degli Studi di Trento and is in great debt to G. Orlandini, W. Leidemann, and N. Barnea for the many instructive conversations and critical comments. The computational resources for this work were in part provided by the RRZE of Universität ErlangenNürnberg. HWG is also grateful for the hospitality of the Institut für Theoretische Physik III at Universität ErlangenNürnberg, of the Institut für Theoretische Physik (T39) at TU München, of the Nuclear Experiment group of the Institut Laue-Langevin (Grenoble, France), and to the organizers and participants of the workshop "Bound States and Resonances in EFTs" at the ECT* (Trento, Italy) for stimulating discussions. Finally, we are indebted to the referees 
for encouraging major improvements and clarifications. This work was supported in part by the CAREER-grant PHY0645498 of the US National Science Foundation and by the US Department of Energy grants DE-FG02-95ER-40907 and DE-FG02-97ER-41019.

\section{Appendix A: Numerical stability: two-nucleon sector}

In the analysis of sect. $\mathrm{V}$, various methods are used to ascertain higher order effects and provide reliable theoretical uncertainties of our NLO calculations. It is therefore imperative to ensure that purely numerical inaccuracies of the variational method are not a significant source of error.

When increasing the model space, two obstacles have to be considered: first, numerical linear dependences amongst the basis vectors; and second, too broad distortion channels. Both issues lead to unstable results and occur especially when the dimension of the model space becomes large. The bulk behavior of the phase shifts in the 4-Helium system was stable in that respect, but the prediction for the ${ }^{3} \mathrm{He}-\mathrm{n}$ spin-zero S-wave scattering length was much more sensitive to the addition of basis vectors. This is a consequence of too broad distortion channels, which is associated with the expansion of the Coulomb functions. We illustrate this point here as a purely numerical issue, anticipating results from sect. V]. All integrals needed to calculate $a_{\lambda j}$ and $b_{\lambda j m}$ have a support only for $R_{j}<R_{\max }$, where $R_{\max }$ is set by the size of the largest fragment. When the Coulomb functions are expanded in Gaussians, this inner region is weighted more at the expense of a less accurate fit for larger separations. This poses no problem if broad Gaussians are used for the distortion channels, i.e., $\omega_{j m}<\omega_{\min }\left(R_{\max }\right)$, because the results are only affected by the values of the Coulomb function in the outer region, $R_{j}>R_{\max }$. In sect. V, potentials are presented which yield relatively weakly bound ${ }^{3} \mathrm{H}$ nuclei with more extended wave functions (see fig. 2). For those interactions, distortion channels with broader width parameters had to be included in order to form the triton cluster inside of the 4-Helium nucleus and reach convergence for the 4-Helium binding energy. If, on the other hand, too many of those broad vectors are included, the phase shifts and especially the scattering length $a_{0}\left({ }^{3} \mathrm{He}-\mathrm{n}\right)$ become unstable.

The NN model space used to fit the LECs (see sect. IV] is a key factor. If the scattering and bound states of a given set of parameters cannot be expanded accurately, the resultant potential will carry a non-negligible model space dependence. To minimize this dependence, a large two-body model space was chosen with a set of width parameters which cover an interval corresponding to the expected extention of the 2-nucleon wave function. Twenty width parameters in the range $\omega_{j m} \in\left[0.001 \mathrm{fm}^{-2} ; 130 \mathrm{fm}^{-2}\right]$ (called set $w_{120}[63]$ ) were used for the NN scattering states, and the same set for the widths $\gamma_{d k}$ in eq. (3) for the S- and D-wave components of the deuteron bound state. Completeness of this set was defined to be sufficient by comparing the results (see table II) to those of another set of 20 parameters, where each inverse width of $w_{120}$ is divided by ten, resulting in broader widths. Hence, all widths of this set $w_{12}$ lie in the interval $\left[0.0001 \mathrm{fm}^{-2} ; 13 \mathrm{fm}^{-2}\right]$. For the triton and 3-Helium wave functions, a previously mentioned set of 224 vectors taken from [54] spanned the model space. It contains all possible couplings with total angular momentum $L \leq 2$ and 142 different width parameters. To model the deuteron fragment in the 4-Helium calculation, its model space was reduced to $6 \mathrm{~S}$-wave and $3 \mathrm{D}$-wave widths for each potential separately, employing a genetic algorithm which optimizes the width parameters of the basis vectors [34]. These sets are named $w_{63}$ in what follows. These optimized deuteron width sets for narrower potentials with larger cutoff values also contained narrower widths, in line with the need to model wave functions with a more complicated short-distance structure.

The expansion of the wave function in terms of Gaussians had significant impact on the choice of the regulator. First, a cutoff momentum space regulator $f(\vec{q})=\exp \left(-\vec{q}^{2} / \Lambda^{2}\right)$, leads to a Gaussian radial dependence as shown below in eq. (8). Hence, all radial dependences of the potential are Gaussians and can directly be implemented into the RRGM. Second, with its functional form fixed, the magnitude of $\Lambda$ fully specifies the regulator and is also influenced by the RRGM. A lower bound is set by the breakdown scale $\Lambda_{b} \approx m_{\pi}$ of $\mathrm{EFT}_{\not}$, since modes which lie in the range of applicability of EFT $\mathrm{ET}_{\star}$ should not be suppressed. On the other hand, an upper bound $\Lambda_{t}$ for the cutoff is set by the numerics of the RRGM. Large cutoff values correspond to narrower Gaussian potentials in coordinate space, and hence the RRGM model space must include narrower Gaussians as well to expand the corresponding, more localized NN bound state accurately, see below. Clearly, $\Lambda$ cannot be chosen considerably larger than the inverse of the narrowest width in which one expands. A LO calculation in the deuteron channel, i.e. $V(\vec{r})=C_{t} \exp \left(-\Lambda^{2} \vec{r}^{2} / 4\right)$, was carried out to illustrate this point. The low-energy constant $C_{t}$ was fitted to reproduce the experimental neutron-proton triplet scattering length $a_{t}^{\exp }$ by numerically solving the two-body Schrödinger equation with MATHEMATICA. The scattering length was calculated from the phase shift at $E_{\mathrm{cm}}=10^{-4} \mathrm{MeV}$, and the resulting coefficient $C_{t}$ was then fed into the RRGM code. The RRGM result for the deuteron binding energy is plotted against $\Lambda$ in fig. 6 , The LO prediction of the effective range expansion, $B(d)=1.4096 \mathrm{MeV}$ from $a_{t}^{\exp }=0.02748 \mathrm{MeV}^{-1}$ [38], is reproduced in both considered model spaces consistently in the RRGM calculation up to the cutoff $\Lambda_{t}$. Above this threshold, $\Lambda_{t}^{w 12} \approx 1.4 \mathrm{GeV}$ and $\Lambda_{t}^{w 120} \approx 3.6 \mathrm{GeV}$, the RRGM solution becomes strongly cutoff-dependent because the respective model space is insufficient to expand a bound state corresponding to such narrow potentials. We explain this as 


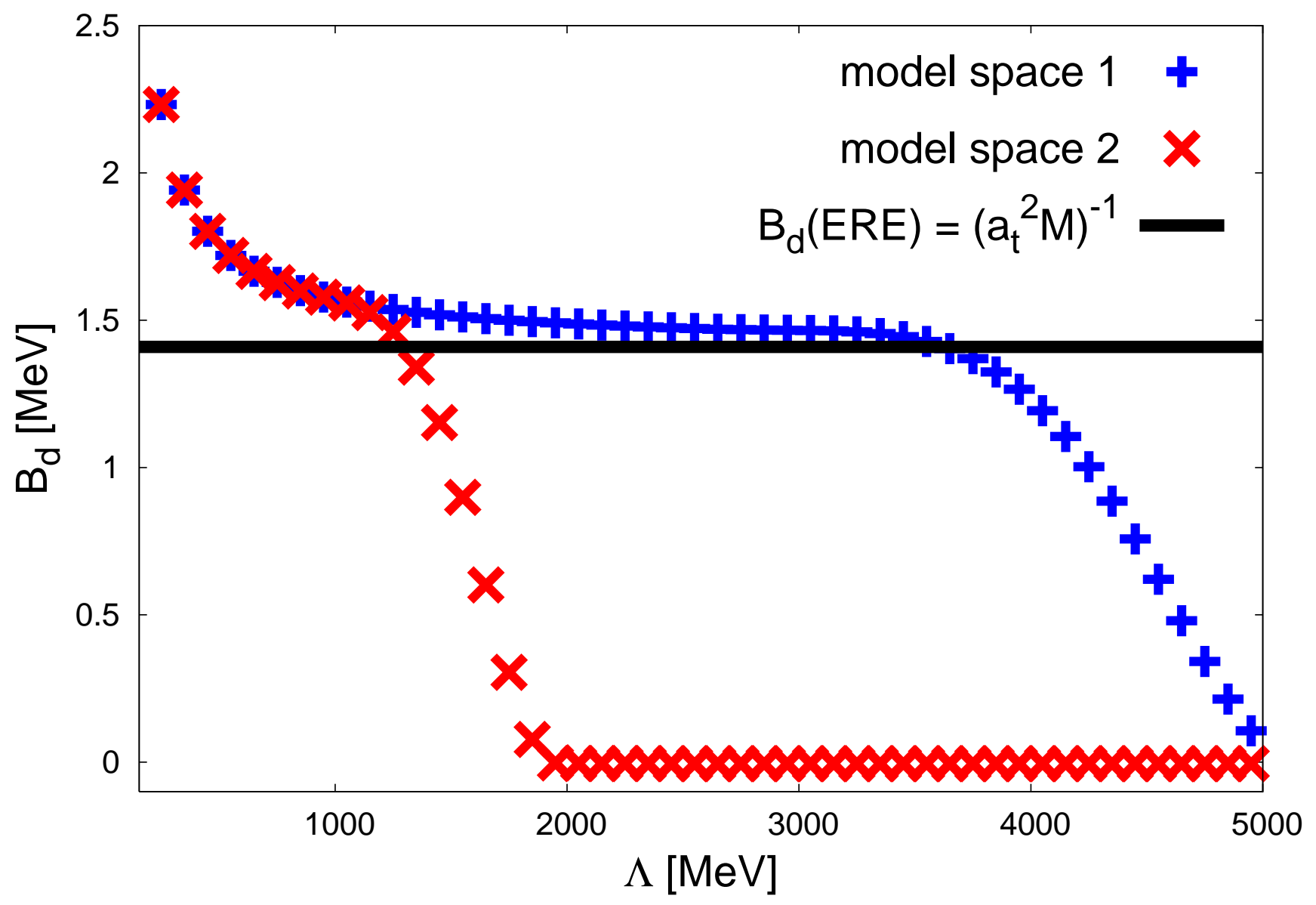

FIG. 6: Binding energy $B_{d}$ of the deuteron in leading-order EFT $\mathrm{ET}_{\not}$ as a function of the Gaussian momentum cutoff $\Lambda$ for two different RRGM model spaces. The space $w_{120}$ contains narrower width parameters, and hence can be used for the potentials corresponding to larger values of $\Lambda$. Using the triplet neutron-proton scattering length as experimental input, the effective range formula predicts $B_{d}=1.41 \mathrm{MeV}$.

follows. The radial ground state wave function reaches a maximum around $r_{p} \approx \Lambda^{-1}$, before decaying exponentially outside the interaction region. Most economically, this peak can be approximated by a sum of two Gaussians with widths comparable to $r_{p}$. For the expansion of the exponential decay, broader Gaussians suffice. If the model space does not contain Gaussians of width $r_{p}$, an approximation of the increasingly steep rise of the wave function at zero accompanying an increasing $\Lambda$ will eventually fail. Hence, bound states can no longer be expanded in the model space for potentials of shorter range than the narrowest Gaussian basis state. In the case shown in fig. 6 relating the narrowest Gaussian widths $\omega_{\max }$ of the two model spaces, $w_{12}$ and $w_{120}$, via the regulator $\exp \left(-\Lambda^{2} \vec{r}^{2} / 4\right)$ to a cutoff $\Lambda_{t}=2 \sqrt{\omega_{\max }}$, one expects the ground state in the respective model space to become unbound for cutoffs larger than $\Lambda_{t}^{w 12} \approx 1.4 \mathrm{GeV}$ and $\Lambda_{t}^{w 120} \approx 4.5 \mathrm{GeV}$. The thresholds suggested in fig. 6 by a drop of the deuteron binding energy are of this expected magnitude. This lead to the conjecture that the $w_{120}$ RRGM model space is appropriate for calculations with cutoff values in a range $150 \mathrm{MeV} \lesssim \Lambda \lesssim 3 \mathrm{GeV}$.

A comparison of the phase shifts $\delta\left({ }^{3} S_{1}\right)$ below $E_{\mathrm{cm}}=10 \mathrm{MeV}$ resulting from an RRGM calculation on the one side, and of a numerical integration of the Schrödinger equation on the other strongly supports this. The results shown in fig. 7 of a potential with a cutoff of $1.5 \mathrm{GeV}$ differ by less than $0.3 \%$. As long as the cutoff was kept below the threshold $\Lambda_{t}$, the relative difference was always found to be of that order. For cutoff values approaching or surpassing $\Lambda_{t}$, the discrepancy increased considerably indicating the predicted failure of the specific RRGM model space.

For the NLO potentials, $\Lambda$ was taken from the interval between $400 \mathrm{MeV}$ and $1 \mathrm{GeV}$, in which $w_{12}$ and $w_{120}$ yielded the same deuteron binding energy. Therefore, it is reasonable to assume that both model spaces are large enough to expand the scattering and bound states of the NLO potentials, so that the model space dependence of the potentials should be minimal. Differences between results using different potentials and short-distance physics do therefore not 


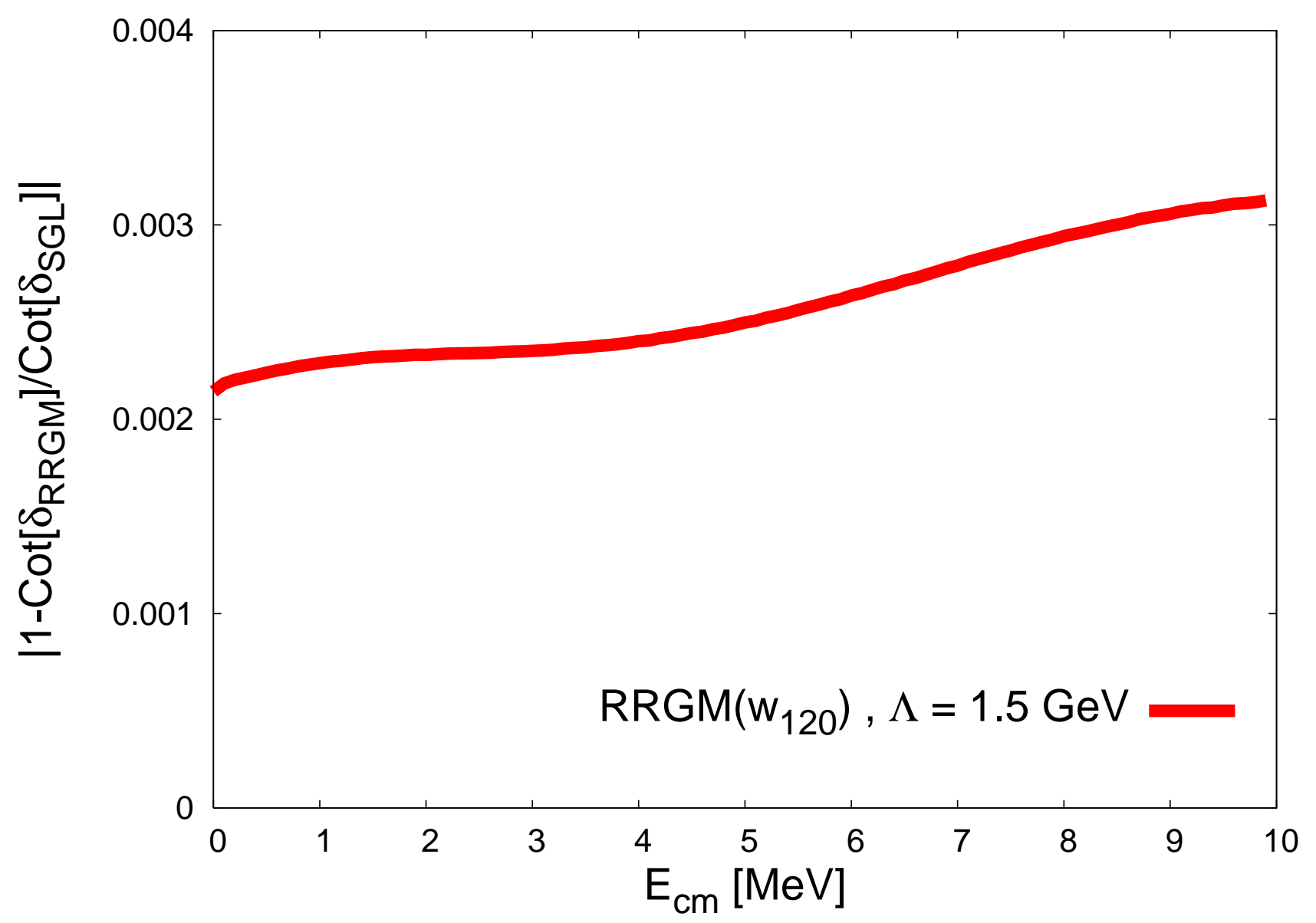

FIG. 7: Relative deviation between the ${ }^{3} S_{1}$ NN phase shift results obtained with the RRGM and a numerical integration of the Schrödinger equation. A leading-order potential with one parameter fit to $a_{t}$ and a cutoff $\Lambda=1.5 \mathrm{GeV}$ was used. Notice the scale on the ordinate.

stem from numerical inaccuracies.

\section{Appendix B: Numerical stability: four-nucleon scattering}

To gauge the size of the error introduced by numerical uncertainties from diagonalizing the Hamiltonian for the calculations with zero $3 \mathrm{NI}, a_{0}\left({ }^{3} \mathrm{He}-\mathrm{n}\right)$ was calculated over a wider range of energies, $E_{\mathrm{cm}} \leq 2 \mathrm{keV}$. Figure 8 shows $\operatorname{Re}\left\{a_{0}\left({ }^{3} \mathrm{He}-\mathrm{n}\right)\right\}$ as a function of the matching energy at which it is calculated for the potential ${ }^{8} V_{\not \star}$. The fluctuations resulting in the band-like shape with a width of about $0.015 \mathrm{fm}$ out of $a_{0}\left({ }^{3} \mathrm{He}-\mathrm{n}\right) \approx 9.415 \mathrm{fm}$ are due to uncertainties associated with the diagonalization of numerically singular matrices. The error due to effective range corrections can be estimated by observing the difference,

$$
\left|\operatorname{Re}\left\{a_{0}(200 \mathrm{eV})\right\}-\operatorname{Re}\left\{a_{0}(2 \mathrm{keV})\right\}\right| \approx 0.01 \mathrm{fm} .
$$

We therefore conclude that the numerical precision of our result is about $1 \%$ and is considerably smaller than the theoretical accuracy of $\sim 10 \%$ attributed to a NLO calculation. For AV18, the same analysis produced stable results with respect to numerical fluctuations, i.e. no visible band as in fig. 8 is found. This is attributed to the model space used for AV18, which was optimized for this potential and purged of states with large but mutually canceling overlap with the ground state. As mentioned at the beginning of this paragraph, this analysis applies to values obtained in an optimized model space only. 


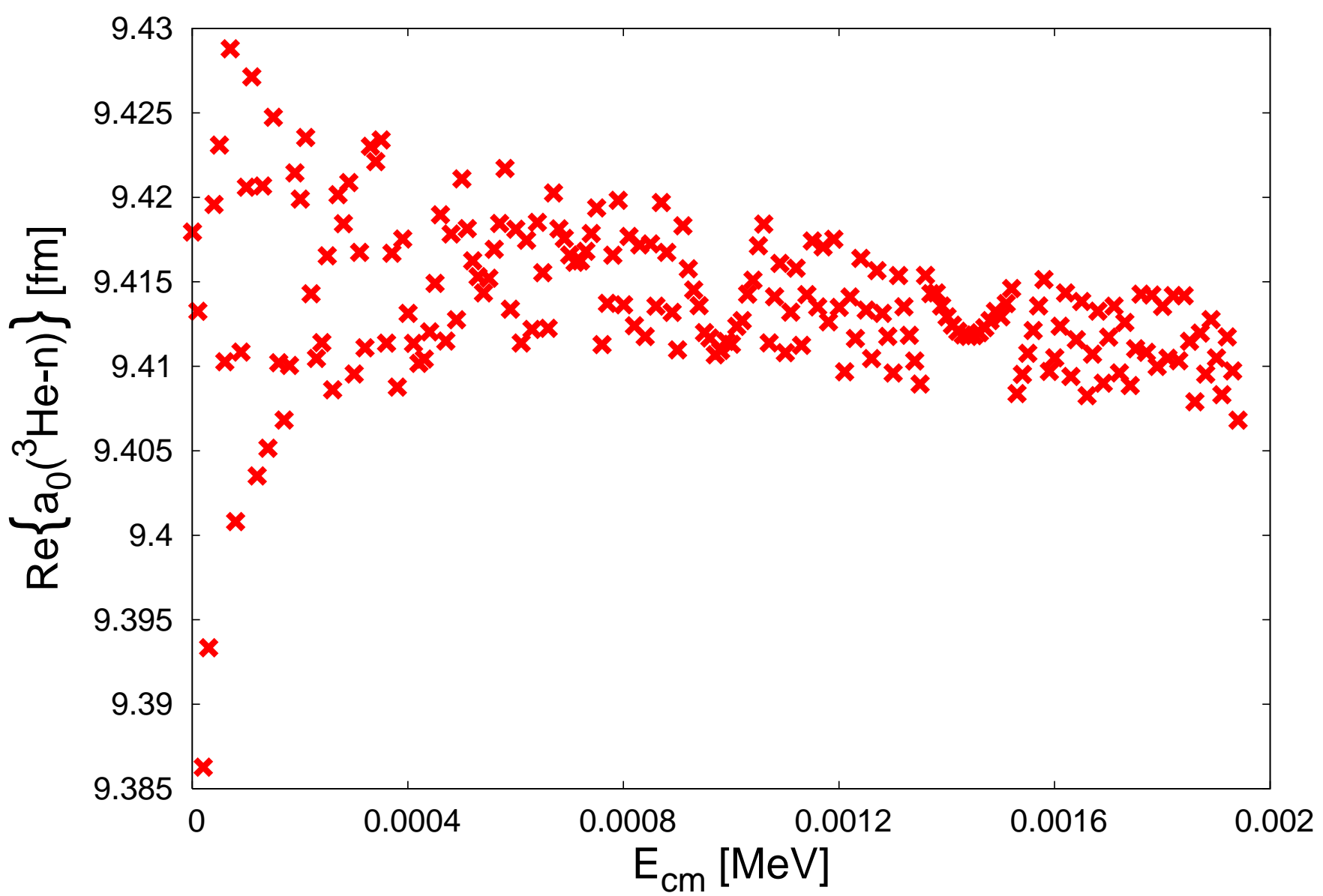

FIG. 8: The real part of the spin singlet, S-wave scattering length for elastic ${ }^{3}$ He-n scattering as a function of the center of mass energy above the ${ }^{3} \mathrm{He}-\mathrm{n}$ threshold at which $a_{0}\left({ }^{3} \mathrm{He}-\mathrm{n}\right)$ is calculated from eq. (18). Notice the scale on the vertical axis. Taking the central value of the band as the actual value, its decrease from $200 \mathrm{eV}$ to $2 \mathrm{keV}$ is due to effective range corrections, while the band is a result of numerical noise. ${ }^{8} V_{\not t}$ was used for this plot, with the other $V_{\not \nless}$ potentials showing similar behavior with respect to the size of the higher order corrections and numerical fluctuations.

\section{Appendix C: Computing time requirements}

In tableIV, the computer time, normalized to one CPU, of the $\mathrm{EFT}_{\text {đ }}$ and the AV18(+UIX) calculations is compared. We display the time used for the build-up of the Hamilton matrix, which surpasses by at least an order of magnitude the time needed for the other stages, namely the calculation of the spin- and coordinate space matrix elements and the diagonalization of the Hamilton matrix. The EFT potentials allow for faster calculations because we choose the regulator of the EFT potentials such that no expansion of the radial dependences is necessary. Hence, only one matrix element is evaluated for a specific operator. For the same operator which has a different radial dependence in AV18, one has to calculate as many matrix elements as Gaussian basis functions are necessary to approximate this dependence accurately. The calculational time per term in the Gaussian expansion is dominated by the operator structure only, and is found to be comparable for AV18 and the EFT potentials. This resembles the already stressed occurrence of all AV18 operators in the EFT potentials considered here. The central 3NI is equally inexpensive with respect to computation time for systems with $A \leq 4$ as the NN potentials. We plan to invest the computer time saved by the convenient choice of the regulator of the $\mathrm{EFT}_{\text {t }}$ interaction in calculating heavier nuclear systems [58]. For those systems, the model space and the number of possible spin and coordinate spaces coupling schemas increases the number of matrix elements to be summed up considerably. Having reduced the number of matrix elements from 
TABLE IV: Runtime comparison between RRGM calculations with EFT and phenomenological potentials. dim(rad-dep) is the number of Gaussians needed to expand the radial dependences, and $\operatorname{dim}(\mathrm{ms})$ is proportional to the dimension of the model space. The parallel computations were performed on IA32 Xeon 2.66 GHz nodes of the RRZE in Erlangen, while for serial code, an E6750 architecture at $2.66 \mathrm{GHz}$ was used.

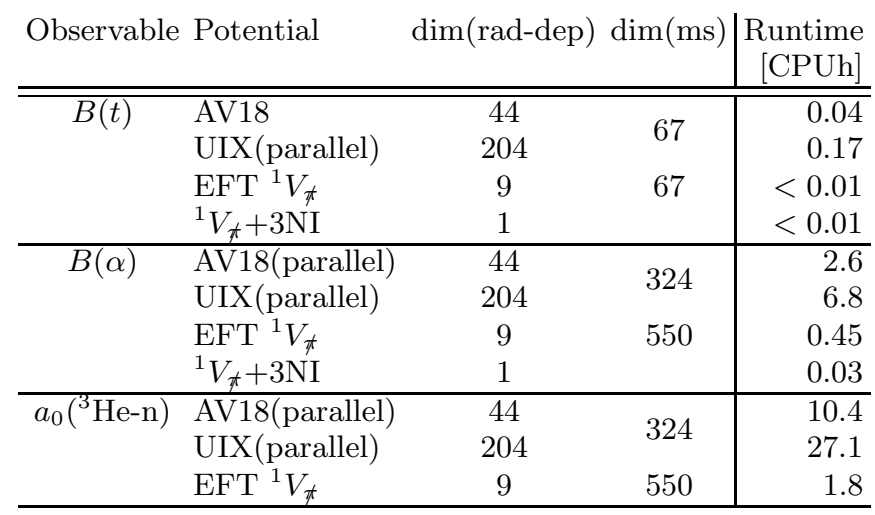

a Gaussian expansion of the potential, therefore, allows for more refined and larger model spaces.

[1] U. van Kolck. Prog. Part. Nucl. Phys., 43:337-418, 1999.

[2] S.R. Beane, P.F. Bedaque, W.C. Haxton, D.R. Phillips, and M.J. Savage. From hadrons to nuclei: Crossing the border. In M. Shifman, editor, At the frontier of particle physics, volume 1, pages 133-269, 2000. arXiv:nucl-th/0008064.

[3] P.F. Bedaque and U. van Kolck. Ann. Rev. Nucl. Part. Sci., 52:339-396, 2002.

[4] L. Platter. Few Body Syst., 46:139-171, 2009.

[5] H.W. Hammer and E. Braaten. Phys. Rept., 428:259-390, 2006.

[6] E. Epelbaum, H.W. Hammer, and U.G. Meißner. Rev. Mod. Phys., 81:1773-1825, 2009.

[7] E. Epelbaum. Nucl. Phys. A, 737:43-51, 2004.

[8] E. Epelbaum. 2010. arXiv:nucl-th/1001.3229.

[9] G. Rupak. Nucl. Phys. A, 678:405, 2000.

[10] L. Platter, H.W. Hammer, and U.G. Meißner. Phys. Lett. B, 607:254-258, 2005.

[11] I. Stetcu, B.R. Barrett, and U. van Kolck. Phys. Lett. B, 653:358-362, 2007.

[12] J.A. Tjon. Phys. Lett. B, 56:217, 1975.

[13] A.C. Phillips. Nucl. Phys. A, 107:209, 1968.

[14] H.W. Hammer and L. Platter. 2010. arXiv:nucl-th/1001.1981.

[15] V. Efimov. Sov. J. Nucl. Phys., 12:589, 1971.

[16] P.F. Bedaque, H.W. Hammer, and U. van Kolck. Phys. Rev. Lett., 82:463-467, 1999.

[17] V.F. Kharchenko. Sov. J. Nucl. Phys., 16:173, 1973.

[18] H.M. Hofmann. In L. S. Ferreira, A. C. Fonseca, and L. Streit, editors, Proceedings of Models and Methods in Few-Body Physics, Lisboa, Portugal, page 243, 1986.

[19] D.R. Phillips. Czech. J. Phys., 52:B49, 2002.

[20] C. Ordonez, L. Ray, and U. van Kolck. Phys. Rev. Lett., 72:1982, 1994.

[21] P.F. Bedaque, H.W. Hammer, and U. van Kolck. Nucl. Phys. A, 646:444-466, 1999.

[22] P.F. Bedaque, G. Rupak, H.W. Grießhammer, and H.W. Hammer. Nucl. Phys. A, 714:589-610, 2003.

[23] H.W. Hammer and T. Mehen. Phys. Lett., B516:353-361, 2001.

[24] L. Platter. Phys. Rev. C, 74:037001, 2006.

[25] L. Platter and D.R. Phillips. FewBodySyst., 40:35-55, 2006.

[26] G.P. Lepage. How to renormalize the Schroedinger equation. 1997. lectures given at 9th Jorge Andre Swieca Summer School: Particles and Fields, Sao Paulo, Brazil, 16-28 Feb 1997. arXiv:nucl-th/9706029.

[27] S. Christlmeier and H.W. Grießhammer. Phys. Rev. C, 77:064001, 2008.

[28] H.W. Grießhammer. Nucl. Phys. A, 744:192-226, 2004.

[29] S. Weinberg. Phys. Lett. B, 251:2, 1990.

[30] S.R. Beane and M.J. Savage. Nucl. Phys. A, 694:511-524, 2001.

[31] D.R. Phillips and T.D. Cohen. Phys. Lett., B390:7-12, 1997.

[32] A.R. Edmonds. Angular Momentum in Quantum Mechanics. Princton University Press, 1996.

[33] R.G. Newton. Scattering Theory of Waves and Particles. Dover Publications, 2002. ch. 14.6.

[34] C. Winkler and H.M. Hofmann. Phys. Rev. C, 55:684-687, 1997.

[35] R.B. Wiringa, V.G.J. Stoks, and R. Schiavilla. Phys. Rev. C, 51:38-51, 1995. 
[36] R. Machleidt, K. Holinde, and C. Elster. Phys. Rept., 149:1-89, 1987.

[37] G.L. Greene, E.G. Kessler, R.D. Deslattes, and H. Börner. Phys. Rev. Lett., 56(8):819-822, Feb 1986.

[38] O. Dumbrajs, R. Koch, H. Pilkuhn, G.C. Oades, H. Behrens, J.J. de Swart, and P. Kroll. Nucl. Phys. B, 216(2):277 - 335, 1983.

[39] V.G.J. Stoks, R.A.M. Klomp, M.C.M. Rentmeester, and J.J. de Swart. Phys. Rev. C, 48:792-815, 1993.

[40] Nijmegen PWA online database, accessed 11/2008, http://nn-online.org/.

[41] L. Platter and H.W. Hammer. Nucl. Phys. A, 766:132-141, 2006.

[42] D.R. Tilley, H.R. Weller, and H.H. Hasan. Nucl. Phys. A, 474(1):1 - 60, 1987.

[43] A.H. Wapstra and G. Audi. Nucl. Phys. A, 432(1):1 - 54, 1985.

[44] G.L. Payne, J.L. Friar, B.F. Gibson, and I.R. Afnan. Phys. Rev. C, 22(2):823-831, 1980.

[45] C.R. Chen, G.L. Payne, J.L. Friar, and B.F. Gibson. Phys. Rev. C, 31(6):2266-2273, 1985.

[46] J.L. Friar, B.F. Gibson, C.R. Chen, and G.L. Payne. Phys. Lett. B, 161:241, 1985.

[47] B.S. Pudliner, V.R. Pandharipande, J. Carlson, S.C. Pieper, and R.B. Wiringa. Phys. Rev. C, 56:1720-1750, 1997.

[48] G.H. Berthold, A. Stadler, and H. Zankel. Phys. Rev. C, 38(1):444-448, 1988.

[49] J.L. Friar, G.L. Payne, and U. van Kolck. Phys. Rev. C, 71:024003, 2005.

[50] G.A. Miller, A.K. Opper, and E.J. Stephenson. Ann. Rev. Nucl. Part. Sci., 56:253-292, 2006.

[51] S. Fiarman and W.E. Meyerhof. Nucl. Phys. A, 206(1):1 - 64, 1973.

[52] J. Kirscher. Diploma thesis FAU Erlangen, 2006. http://theorie3.physik.uni-erlangen.de/theses/data/Dip-2006-01.pdf.

[53] A. Deltuva and A.C. Fonseca. Phys. Rev. C, 75:014005, 2007.

[54] H.M. Hofmann and G.M. Hale. Phys. Rev. C, 77:044002, 2008.

[55] S.C. Pieper, V.R. Pandharipande, R.B. Wiringa, and J. Carlson. Phys. Rev. C, 64:014001, 2001.

[56] P.R. Huffman et al. Phys. Rev. C, 70:014004, 2004.

[57] O. Zimmer, G. Ehlers, B. Farago, H. Humblot, W. Ketter, and R. Scherm. EPJdirect, A1:1-28, 2002.

[58] J. Kirscher, H.W. Grießhammer, and H.M. Hofmann. forthcoming.

[59] C.A. Bertulani, H.W. Hammer, and U. Van Kolck. Nucl. Phys. A, 712:37-58, 2002.

[60] T. Shima et al. Phys. Rev. C, 72:044004, 2005.

[61] S. Quaglioni, W. Leidemann, G. Orlandini, N. Barnea, and V.D. Efros. Phys. Rev. C, 69:044002, 2004.

[62] W. Sandhas, W. Schadow, G. Ellerkmann, L.L. Howell, and S.A. Sofianos. Nucl. Phys. A, 631:210c-229c, 1998.

[63] C. Reiss and H.M. Hofmann. Nucl. Phys. A, 716:107-119, 2003.

[64] We thank W. Glöckle and D. R. Phillips for remarks on this point, which helped to sharpen our view on this issue. 